Article

\title{
Genome-Wide Linkage Mapping of Quantitative Trait Loci for Late-Season Physiological and Agronomic Traits in Spring Wheat under Irrigated Conditions
}

\author{
Yuxiu Liu ${ }^{1,2}$, Rui Wang ${ }^{1}\left(\mathbb{D}\right.$, Yin-gang $\mathrm{Hu}^{2,3}$ and Jianli Chen ${ }^{1, *}$ \\ 1 Department of Plant, Soil, and Entomological Sciences, University of Idaho, 1691 S 2700 W, \\ Aberdeen, ID 83210, USA; liuyuxiu2007@126.com (Y.L.); ruiw@uidaho.edu (R.W.) \\ 2 State Key Laboratory of Crop Stress Biology for Arid Areas, College of Agronomy, \\ Northwest A\&F University, Yangling 712100, Shaanxi, China; huyingang@126.com \\ 3 Institute of Water Saving Agriculture in Arid Regions of China, Yangling 712100, Shaanxi, China \\ * Correspondence: jchen@uidaho.edu; Tel.: +1-208-397-4162 (ext. 229)
}

Received: 28 March 2018; Accepted: 21 April 2018; Published: 25 April 2018

\begin{abstract}
Many late-season physiological traits affect grain yield in wheat, either directly or indirectly. However, information on the genetic control of yield-related traits is still limited. In this study, we aimed to identify quantitative trait loci (QTL) for canopy temperature and chlorophyll content index during anthesis (CTa and CCIa, respectively), the mid grain-filling stage (CTg1 and CCIg1, respectively), and the late grain-filling stage (CTg2 and CCIg2, respectively) as well as for plant height (PH), thousand kernels weight (TKW), and grain yield (GY) using genome-wide linkage mapping. To this end, a double haploid population derived from a cross between two high yielding wheat cultivars, UI Platinum and SY Capstone, was phenotyped in four irrigated environments and genotyped using the wheat $90 \mathrm{~K}$ iSelect platform and simple sequence repeats. The genotypic data were used to construct a high-density genetic map of 43 linkage groups (LGs) with a total length of $3594.0 \mathrm{~cm}$ and a marker density of $0.37 \mathrm{~cm}$. A total of 116 QTL for all nine traits was detected on 33 LGs, spreading to all wheat chromosomes, except for Chr. 7D. Of these, six QTL (CTa.ui-4B.1, Q.CTg1.ui-5B-2.1, Q.CTg2.ui-6B.1, Q.PH.ui-6A-2.1, Q.TKW.ui-2D-1, and Q.GY.ui-6B) were consistently detected in more than three irrigated environments, called as stable QTL. Additionally, we identified 26 QTL clusters for more than two traits, of which the top four were located on Chromosomes 4A-1, 1B-1, 5B-2, and 2D-1. Overall, the stable QTL significantly related with grain yield, QTL clusters, and linked molecular markers identified in this study, may be useful in marker-assisted selection in early generation and early growth stage for grain yield improvement.
\end{abstract}

Keywords: linkage mapping; QTL; 90K SNP; canopy temperature; chlorophyll content index; plant height; grain yield

\section{Introduction}

Wheat (Triticum aestivum L.) is one of the most important food crops worldwide [1] since it is a valuable source of carbohydrates and protein suitable for human consumption [2]. However, wheat production is negatively affected by the gradual reduction of arable land area due to climate change as well as biotic and abiotic stresses [3-6]. Therefore, further improvement in grain yield (GY) is necessary to meet the current and future demand.

Canopy temperature (CT) or CT depression and chlorophyll content index (CCI) or SPAD value of leaf chlorophyll content are important physiological traits significantly associated with GY in wheat [7-11]. $\mathrm{CT}$ is considered an indicator of the transpiration level, whereas the chlorophyll content of flag leaf is 
used for the indirect estimation of photosynthesis capacity [12]. A high chlorophyll content in the flag leaf during post-anthesis positively affects yield stability by extending photosynthesis under a wide range of conditions [13]. Agronomic traits, such as plant height (PH) and thousand kernels weight (TKW), are also closely related to GY $[8,11]$. Therefore, it would be useful to elucidate the genetic control of late-season physiological traits that are closely related to GY in order to further improve wheat yield.

Molecular techniques have accelerated progress in plant breeding programs [14]. Of these, quantitative trait locus (QTL) mapping that is carried out to detect potential chromosome regions associated with important traits using molecular markers [15] has been proved to be an effective way to understand the genetic architecture of physiological and yield-related traits in crops [16]. In wheat, QTL mapping has been extensively conducted for PH, TKW, and GY [1,9,11,17-24] as well as for CT (or CT depression) and CCI (or SPAD), since these two traits are significantly correlated with GY and its components [9-11]. Some QTL for CT (or CT depression) and CCI (or SPAD) have been identified during anthesis or at $10 \mathrm{~d}$ (days) post-anthesis using different mapping populations and considered as potential tools for improving wheat yield under different stress environments [7,9-11,21,25-27]. QTL for CT during the vegetative and grain-filling stages have been identified on Chromosomes (Chr.) 1B, 2B, 3B, and 7A across different environments, whereas one QTL for CT during the vegetative stage has been identified on Chr. 4A [28]. Previous study reported QTL for CT during the vegetative ( $25 \mathrm{~d}$ after emergence) and grain-filling stages on Chr. 3A, 3BS, 3BL, 5B, and 7A under irrigated, heat, and drought conditions [21]. QTL for CT depression during anthesis have been mapped on Chr. 2AL, 3BL, 4BS, 5BS, and 6BL, whereas QTL for CT depression at $10 \mathrm{~d}$ post-anthesis on Chr. 4AS, 4BS, and 5BS [10]. QTL for SPAD at $0 \mathrm{~d}, 7 \mathrm{~d}$, and $14 \mathrm{~d}$ post-anthesis have been detected on Chr. 1A, 1B, 1D, 2B, 3B, 4D, 6B, and 7B under unstressed conditions [29]; on Chr. 1A, 2B, 3A, 4A, $4 \mathrm{D}, 5 \mathrm{~B}$, and $6 \mathrm{~A}$ at $0 \mathrm{~d}, 7 \mathrm{~d}$, and $14 \mathrm{~d}$ post-anthesis under high-light stress conditions [7]; on Chr. 3D and 7A at the seeding stage under salt stress conditions [26]; on Chr. 7A during anthesis under different nitrogen and water conditions [25]; and on $\mathrm{Chr}$. 1B and on 1A, 1D, 3A, 3B, and 5A under heat and drought conditions [28]. Previous study reported QTL for SPAD during anthesis on Chr. 2AS, 2AL (2), 2DS, 2DL, 3AS, 4AL, 4DS, $5 \mathrm{AS}$, and 5AL, and at $10 \mathrm{~d}$ post-anthesis on Chr. 2AL (2), 2BS, 2D, 5AL, 5BL, 6AS, and 7A [11].

However, only a few QTL for CT and CCI have been reported during the late growth stages under irrigated conditions. Additionally, most studies used a limited number of markers and resulted in QTL with relatively large genetic distances, which are not appropriate for marker-assisted selection (MAS). The high-density single nucleotide polymorphism (SNP) technology is an efficient tool for QTL detection, since it allows the construction of high-density linkage maps [30,31]. SNP makers are co-dominant, abundant, and evenly distributed along the genome [32,33]. In wheat, the $9 \mathrm{~K}$ and $90 \mathrm{~K}$ iSelect SNP arrays have been successfully applied for the QTL mapping of yield and yield-related traits [10,11,23,31,34,35].

Here, we aimed to identify QTL for CT and CCI during anthesis, the early grain-filling stage, and the middle grain-filling stage as well as for $\mathrm{PH}, \mathrm{TKW}$, and GY in a double haploid (DH) population that phenotyped under irrigated conditions and genotyped using genome-wide high-density SNP markers combined with simple sequence repeats (SSRs).

\section{Materials and Methods}

\subsection{Plant Materials and Field Experiments}

In this study, we used a mapping population, consisting of 110 doubled haploid (DH) lines that derived from a cross between two spring wheat cultivars, UI Platinum and SY Capstone. The former cultivar (PI 672533) was developed by the Idaho Agricultural Experimental Station and released in 2014 [36], whereas the latter was developed by Syngenta Cereals and released in 2011 [37]. The DH lines were created from $\mathrm{F}_{1}$ under service from Heartland Plant Innovation using wheat by maize hybridization [38].

Four trials were performed in Aberdeen, Idaho, USA ( $42.96^{\circ} \mathrm{N}, 112.83^{\circ} \mathrm{W}$; elevation $\left.1342 \mathrm{~m}\right)$ in the 2014/2015 and 2015/2016 cropping seasons under irrigated conditions (AB15E1, AB15E2, AB15E3, and AB16E4). A detailed description of all environments is provided in Table 1. The precipitation of four trails during the growing season was $217.4 \mathrm{~mm}, 217.4 \mathrm{~mm}, 217.4 \mathrm{~mm}$, and $249.0 \mathrm{~mm}$, respectively, 
and irrigation was applied from May to July with a total amount of $362.7 \mathrm{~mm}, 373.4 \mathrm{~mm}, 384.0 \mathrm{~mm}$ and $453.4 \mathrm{~mm}$, respectively, making total moisture $580.1 \mathrm{~mm}, 590.8 \mathrm{~mm}, 601.4 \mathrm{~mm}$ and $702.4 \mathrm{~mm}$, using aluminum sprinkler pipes. In AB15E1, and AB15E2, 120 entries were arranged in an augmented design. 120 replicated DH lines were assigned at random to four blocks, while two parents as checks were replicated, being randomly assigned to plots within each of the four blocks, therefore, each sub-block comprised 28 un-replicated DH lines and the two cultivar checks. In AB15E3 and AB16E4, the DH and parental lines were arranged in a randomized complete block design with two replications. Each plot included seven rows ( $3.0 \mathrm{~m}$ in length, $1.5 \mathrm{~m}$ in width, $0.25 \mathrm{~m}$ in row spacing). The sowing density was 0.48 million seeds per ha at each trial. Fertilization and weeding were applied, when necessary, to achieve the optimal cultivation conditions. A wheat border was planted to minimize the edge effect in each trial.

Table 1. Rainfall and irrigation ( $\mathrm{mm}$ ) during the two wheat-growing seasons for the 4 trials.

\begin{tabular}{|c|c|c|c|c|c|c|c|c|c|c|c|c|c|}
\hline \multirow{2}{*}{ Trials } & \multirow{2}{*}{ Sowing Date } & \multirow{2}{*}{$\begin{array}{c}\text { September-March } \\
\text { R }\end{array}$} & \multicolumn{2}{|c|}{ April } & \multicolumn{2}{|c|}{ May } & \multicolumn{2}{|c|}{ June } & \multicolumn{2}{|c|}{ July } & \multicolumn{2}{|c|}{ August } & \multirow{2}{*}{ Total } \\
\hline & & & $\mathbf{R}$ & IRR & $\mathbf{R}$ & IRR & $\mathbf{R}$ & IRR & $\mathbf{R}$ & IRR & $\mathbf{R}$ & IRR & \\
\hline 15ABE1 & 20 March 2015 & 92.4 & 2.5 & 42.7 & 83.6 & 53.3 & 19.8 & 181.4 & 19.1 & 85.3 & - & / & 580.1 \\
\hline 15ABE2 & 26 March 2015 & 92.4 & 2.5 & 42.7 & 83.6 & 53.3 & 19.8 & 181.4 & 19.1 & 96.0 & - & / & 590.8 \\
\hline $15 \mathrm{ABE} 3$ & 1 April 2015 & 92.4 & 2.5 & 42.7 & 83.6 & 53.3 & 19.8 & 192.0 & 19.1 & 96.0 & - & / & 601.4 \\
\hline 16ABE4 & 8 April 2016 & 165.4 & 43.2 & / & 36.8 & 74.7 & 0.8 & 218.7 & 2.8 & 160.0 & 0 & / & 702.4 \\
\hline
\end{tabular}

15ABE1, 15ABE2, 15ABE3: trial 1, 2 and 3 in Aberdeen in 2015, respectively; 16ABE4, trial 4 in Aberdeen in 2016;

$\mathrm{R}$, rainfall; IRR, irrigation water; -, not included; /, no irrigation.

\subsection{Phenotypic Evaluation}

$\mathrm{CT}, \mathrm{CCI}, \mathrm{PH}, \mathrm{TKW}$, and GY were recorded in all four environments in two seasons (AB15E1, $\mathrm{AB} 15 \mathrm{E} 2, \mathrm{AB} 15 \mathrm{E} 3$, and $\mathrm{AB} 16 \mathrm{E} 4) . \mathrm{CT}\left({ }^{\circ} \mathrm{C}\right)$ was measured using an infrared thermometer (IRtec MicroRay HVAC; Langhorne, PA) from noon to 2:00 PM on clear and non-windy days. The CCI of flag leaves was measured from five randomly selected fertile plants using a portable chlorophyll content meter (CCM-200; Opti-Sciences, Hudson, NH, USA). CT and CCI were measured during anthesis (Feekes 10.5.2; CTa and CCIa, respectively), the mid grain-filling stage (Feekes 11.1; CTg1 and CCIg1, respectively), and the late grain-filling stages (Feekes 11.2; CTg2 and CCIg2, respectively) using the Feekes growth scale [39].

$\mathrm{PH}(\mathrm{cm})$ was measured from the soil surface to the tip of the spike (awns excluded) at the maturity stage (Feekes 11.3-11.4) [39]. Plots were harvested by a Wintersteiger Classic small plot combine (Wintersteiger; Salt Lake City, UT, USA) equipped with a Harvest Master weighing system (Juniper Systems; Logan, UT, USA). After harvest, GY was determined as grain weight extrapolated at the $12 \%$ moisture content and expressed in $\mathrm{tha}^{-1}$. TKW (g) was recorded using the single-kernel characteristics system (SKCS 4100; Perten Instruments, Springfield, IL, USA).

\subsection{Phenotypic Data Analysis}

Phenotypic data analysis, including mean, genotype variance $\left(\delta_{g}^{2}\right)$, error variance $\left(\delta_{e}^{2}\right)$, correlation analysis between parameters, and broad-sense heritability $\left(H_{B}^{2}\right)$, was conducted using JMP 8.0 (SAS Institute, Cary, NC, USA). The adjusted means of each trait measured in AB15E1 and AB15E2 were estimated for block differences which were measured by the cultivar checks [40]. The analysis of variance was performed with PROC GLM, in which genotypes were treated as fixed effects and environments and the interaction of genotypes and environments and blocks nested in environments were all treated as random effects [40]. The inverse of the variance of the individual environments were treated as weights. $H_{B}^{2}$ was estimated in all environments as follows:

$$
H_{B}^{2}=\sigma_{g}^{2} /\left(\sigma_{g}^{2}+\sigma_{g e}^{2} / r+\sigma_{\varepsilon}^{2} / e r\right),
$$

where $\delta_{g}^{2}$ is the genetic variance, $\sigma_{g e}^{2}$ is the genetic $\times$ environment interaction variance, $\delta_{\varepsilon}^{2}$ is the error variance, $r$ is the replicates per environment, and $e$ is the number of environments. 


\subsection{SNP Genotyping and Molecular Marker Analysis}

Total genomic DNA was extracted from young leaves of the DH and parental lines using the method as described by [41]. DNA concentration was estimated using a Nanodrop ND-1000 Spectrophotometer (Nanodrop; Wilmington, DE, USA) and then, adjusted to $80 \mathrm{ng} \mu \mathrm{L}^{-1}$ for SNP assays and molecular marker analysis.

The DH and parental lines were genotyped at the USDA-ARS Small Grains Genotyping Laboratory, Fargo, ND, USA, using the Illumina $90 \mathrm{~K}$ iSelect SNP array [31]. Genotype calling and SNP clustering were conducted using GenomeStudio 2011 with the polyploid clustering V1-0 (Illumina, San Diego, CA, USA). $\mathrm{SNP}$ names were designated as "IWB" followed by an index number.

A total of 300 SSRs was selected for genotyping the parental lines as described by [42]. Molecular markers for dwarfing genes ( $R h t-B 1 b$ and $R h t-D 1 b$ ) were used for confirming the association with PH $[43,44]$.

\subsection{Linkage Map Construction and QTL Analysis}

SNP markers were filtered for monomorphism, high frequency of missing values $(\geq 10 \%)$, or segregation distortion $(\geq 0.35)$. The genetic linkage map was constructed using JMP Genomics 8.0 (SAS Institute) as described by [45] and [46]. The initial number of linkage groups (LGs) was identified using interactive hierarchical clustering and K-means clustering (automated radius K-means) by reducing the number of markers in the recombination and LG function. Markers were ordered on each LG using the Kosambi mapping function and the accelerated map order optimization algorithm in the linkage map order function. LGs were split when the genetic distance between adjacent markers was higher than $35 \mathrm{~cm}$ by default.

QTL analysis was performed for each trait within and across four environments by composite interval mapping (CIM) using JMP Genomics 8.0 [46]. CIM was used to find QTL with an expectation maximization (EM) algorithm threshold of 2.5. Genetic distance between markers was calculated in $\mathrm{cm}$. The contribution of SY and UI toward higher trait values was indicated by positive and negative signs of the estimates for additive QTL effects, respectively. The proportion of phenotypic variation $\left(R^{2}\right)$ for each QTL was determined by the square of the partial correlation coefficient.

The final map was constructed using the linkage map viewer function of JMP Genomics, and LGs were assigned to chromosomes based on the $90 \mathrm{~K}$ consensus map as described by [31]. Each linkage map was named according to the wheat chromosome followed by a number. A QTL was characterized as stable when it was identified in at least three or four environments.

\section{Results}

\subsection{Phenotypic Evaluation}

The analysis of variance showed that the effect of genotype was significant for all traits across environments, except for CTa and CTg1(across 15ABE3 and 16ABE4) $(p<0.05)$, whereas the effect of environment and that of genotype by environment were significant for CTa, CTg2, CCIa, CCIg2 and GY across 15ABE1 and 15ABE2 as well as CCIg2, TKW and GY across 15ABE3 and 16ABE4 $(p<0.05)$ (Table 2). The phenotypic performance of DH and parental lines indicated that UI and SY were significantly different for CCI and TKW in all the environments (Table S1); UI had markedly higher TKW, whereas SY had higher CCIa, CCIg1, and CCIg2. The mean for most traits of DH lines was near to the mid-parental value. The range of variation for each trait of $\mathrm{DH}$ lines showed transgressive segregation in both directions across the environments, demonstrating that positive alleles were present from both parental lines. All traits showed continuous frequency distribution within and across the environments, indicating that they were under polygenic control (Figure S1). The $H_{B}{ }^{2}$ was the highest for TKW ( 0.81 across 15ABE1 and 15ABE2, 0.72 across 15ABE3 and 16ABE4) and $\mathrm{PH}$ (0.77 across 15ABE1 and 15ABE2, 0.75 across $15 \mathrm{ABE} 3$ and 16ABE4), indicating that both traits were stable and mainly affected by the genotype, whereas the $H_{B}{ }^{2}$ was the lowest for $\mathrm{CTa}, \mathrm{CTg} 1$, and $\mathrm{CTg} 2$, suggesting that they were greatly affected by the environment. 
Table 2. Analysis of variance (ANOVA) and broad-sense heritability $\left(H_{B}{ }^{2}\right)$ for the traits measured in four environments (110 doubled haploid (DH) lines and two cultivar checks in 15ABE1 and 15ABE2, two replications in 15ABE3 and 16ABE4).

\begin{tabular}{|c|c|c|c|c|c|c|c|c|c|c|}
\hline \multirow{2}{*}{ SV } & \multirow{2}{*}{ DF } & \multicolumn{2}{|c|}{ MS } & \multicolumn{2}{|c|}{ Across 15ABE1 and 15ABE2 } & \multirow{2}{*}{ DF } & \multicolumn{2}{|c|}{ MS } & \multicolumn{2}{|c|}{ Across 15ABE3 and 16ABE4 } \\
\hline & & 15ABE1 & 15ABE2 & DF & MS & & 15ABE3 & 16ABE4 & DF & MS \\
\hline \multicolumn{11}{|l|}{$\mathrm{CTa}$} \\
\hline Block/Replication & 3 & 0.08 & 0.36 & & & & 0.79 & $163.57^{* * * *}$ & & \\
\hline Environment (E) & & & & 1 & $4.46^{* *}$ & & & & 1 & $4531.88^{* * * *}$ \\
\hline$G \times E$ & & & & 111 & $1.31^{* *}$ & & & & 109 & 0.67 \\
\hline Error & 3 & 0.09 & 0.51 & 12 & 0.26 & & 0.56 & 1.27 & 220 & 1.65 \\
\hline$H_{B}^{2}$ & & & & & 0.52 & & & & & 0.38 \\
\hline Genotype $(\mathrm{G})$ & 111 & $1.89 *$ & 0.15 & 111 & $1.84^{*}$ & & 0.60 & 2.51 & 109 & 1.70 \\
\hline Environment (E) & & & & 1 & 1.16 & & & & 1 & 0.57 \\
\hline$G \times E$ & & & & 111 & $1.98 *$ & & & & 109 & 1.41 \\
\hline Error & 3 & 0.15 & 0.06 & 12 & 0.60 & & 0.50 & 2.38 & 220 & 1.81 \\
\hline$H_{B}^{2}$ & & & & & 0.45 & & & & & 0.42 \\
\hline \multicolumn{11}{|l|}{ CTg2 } \\
\hline$H_{B}^{2}$ & & & & & 0.49 & & & & & 0.49 \\
\hline \multicolumn{11}{|l|}{ CCIa } \\
\hline Block/Replication & 3 & $5.31 *$ & 1.92 & & & & 6.19 & $78.00 *$ & & \\
\hline Genotype $(G)$ & 111 & $30.45^{* *}$ & $19.18 *$ & 111 & $27.36^{* * * *}$ & & $18.16^{* *}$ & $28.15^{* * *}$ & 109 & $32.93 * * * *$ \\
\hline Environment (E) & & & & 1 & $340.08^{* * * *}$ & & & & 1 & 2.11 \\
\hline$G \times E$ & & & & 111 & $23.74^{* * * *}$ & & & & 109 & 13.38 \\
\hline Error & 3 & 0.43 & 1.05 & 12 & 2.18 & & 11.52 & 14.89 & 220 & 13.47 \\
\hline$H_{B}^{2}$ & & & & & 0.52 & & & & & 0.62 \\
\hline \multicolumn{11}{|l|}{ CCIg1 } \\
\hline Block/Replication & 3 & 8.44 & 2.81 & & & & 2.86 & 61.16 & & \\
\hline Genotype (G) & 111 & 36.31 & 26.20 & 111 & $38.21 * * *$ & & $37.24 *$ & $41.32 * * * *$ & 109 & $56.37 * * * *$ \\
\hline
\end{tabular}


Table 2. Cont.

\begin{tabular}{|c|c|c|c|c|c|c|c|c|c|c|}
\hline \multirow{2}{*}{ SV } & \multirow{2}{*}{ DF } & \multicolumn{2}{|c|}{ MS } & \multicolumn{2}{|c|}{ Across 15ABE1 and 15ABE2 } & \multirow{2}{*}{ DF } & \multicolumn{2}{|c|}{ MS } & \multicolumn{2}{|c|}{ Across 15ABE3 and 16ABE4 } \\
\hline & & 15ABE1 & 15ABE2 & DF & MS & & 15ABE3 & 16ABE4 & DF & MS \\
\hline Environment (E) & & & & 1 & 16.47 & & & & 1 & $1600.20^{* * * *}$ \\
\hline$G \times E$ & & & & 111 & $25.31 * *$ & & & & 109 & 22.19 \\
\hline$H_{B}^{2}$ & & & & & 0.57 & & & & & 0.63 \\
\hline \multicolumn{11}{|l|}{ CCIg2 } \\
\hline Block/Replication & 3 & 3.79 & 6.41 & & & & 22.66 & 54.80 & & \\
\hline $\mathrm{G} \times \mathrm{E}$ & & & & 111 & $20.42 *$ & & & & 109 & $19.46^{*}$ \\
\hline Error & 3 & 12.25 & 4.69 & 12 & 6.79 & & 13.72 & 16.05 & 220 & 15.10 \\
\hline$H_{B}^{2}$ & & & & & 0.57 & & & & & 0.63 \\
\hline \multicolumn{11}{|l|}{ PH } \\
\hline Block/Replication & 3 & 1.38 & 2.61 & & & & $708.13^{* * * *}$ & 3.07 & & \\
\hline Genotype (G) & 111 & 22.31 & 27.57 & 111 & $43.20 * * *$ & & $42.56 * * * *$ & $55.95^{* * * *}$ & 109 & $81.12 * * * *$ \\
\hline Environment (E) & & & & 1 & 0.02 & & & & 1 & $6763.57^{* * * *}$ \\
\hline Block/Replication & 3 & 4.07 & 2.11 & & & & $47.48^{* *}$ & $38.89 *$ & & \\
\hline Genotype $(\mathrm{G})$ & 111 & 13.74 & 9.87 & 111 & $21.21 * * *$ & & $18.79^{* * * *}$ & $19.60^{* * * *}$ & 109 & $29.85^{* * * *}$ \\
\hline Environment (E) & & & & 1 & 7.44 & & & & 1 & $2480.45^{* * * *}$ \\
\hline$G \times E$ & & & & 111 & 3.05 & & & & 109 & $8.53 *$ \\
\hline Error & 3 & 5.64 & 3.28 & 12 & 3.78 & & 4.97 & 6.23 & 220 & 5.95 \\
\hline$H_{B}^{2}$ & & & & & 0.81 & & & & & 0.72 \\
\hline \multicolumn{11}{|l|}{ GY } \\
\hline Block/Replication & 3 & 0.20 & 0.13 & & & & $5.06^{* * * *}$ & 1.80 & & \\
\hline Genotype (G) & 111 & 0.40 & 0.53 & 111 & $0.71^{* *}$ & & $0.72^{* * * *}$ & $1.59^{* * * *}$ & 109 & $1.67^{* * * *}$ \\
\hline Environment (E) & & & & 1 & $66.28^{* * * *}$ & & & & 1 & $6.16^{* * *}$ \\
\hline$G \times E$ & & & & 111 & $0.32 *$ & & & & 109 & $0.64^{* *}$ \\
\hline Error & 3 & 0.11 & 0.08 & 12 & 0.13 & & 0.26 & 0.55 & 220 & 0.43 \\
\hline$H_{B}^{2}$ & & & & & 0.65 & & & & & 0.66 \\
\hline
\end{tabular}

15ABE1, 15ABE2, 15ABE3: trial 1, 2 and 3 in Aberdeen in 2015, respectively; 16ABE4, trial 4 in Aberdeen in 2016; SV, Source of variation; DF, degree of freedom; MS, Mean square, CTa, CTg1, CTg2, canopy temperature during anthesis, mid- and late grain-filling stage, respectively; CCIa, CCIg1, CCIg2, chlorophyll content index during anthesis, mid- and late grain-filling stage, respectively; PH, plant height; TKW, thousand kernels weight; GY, grain yield; $* *, p<0.01 ; * *, p<0.01 ; * * *, p<0.001 ; * * *, p<0.0001$. 


\subsection{Correlation between Traits}

Pearson's correlation between traits was analyzed across environments (Table 3). CTa, CTg1, and CTg2 were significantly negatively correlated with GY $(r=-0.72-0.89, p<0.0001)$, whereas CCIa, PH, and TKW were significantly positively correlated with GY $(r=0.45-0.87, p<0.001)$. CTa, CTg1, and CTg2 were significantly negatively correlated with CCIa, PH, and TKW $(r=-0.30-0.77, p<0.01)$, whereas CCla was significantly positively correlated with PH and TKW $(r=0.33-0.37, p<0.0001)$. PH was significantly positively correlated with TKW $(r=0.71, p<0.0001)$. Significant positive correlations were also found between CTa, CTg1, and CTg2 $(r=0.50-0.83, p<0.0001)$ as well as between CCIa, CCIg1, and CCIg2 $(r=0.33-0.65, p<0.0001)$.

Table 3. Pearson's correlations between phenotypic traits measured in DH population from UI Platinum/SY Capstone over four environments.

\begin{tabular}{ccccccccc}
\hline & CTa & CTg1 & CTg2 & CCIa & CCIg1 & CCIg2 & PH & TKW \\
\hline CTg1 & $0.50^{* * * *}$ & & & & & & & \\
CTg2 & $0.61^{* * * *}$ & $0.83^{* * * *}$ & & & & & & \\
CCIa & $-0.30^{* *}$ & $-0.34^{* *}$ & $-0.44^{* * * *}$ & & & & & \\
CCIg1 & $0.24^{*}$ & 0.11 & 0.08 & $0.33^{* * * *}$ & & & & \\
CCIg2 & 0.02 & -0.05 & -0.13 & $0.60^{* * * *}$ & $0.65^{* * * *}$ & & & \\
PH & $-0.77^{* * * *}$ & $-0.60^{* * * *}$ & $-0.71^{* * * *}$ & $0.37^{* * * *}$ & $-0.11^{*}$ & 0.09 & & \\
TKW & $-0.71^{* * * *}$ & $-0.55^{* * * *}$ & $-0.62^{* * * *}$ & $0.33^{* * * *}$ & $-0.12^{*}$ & 0.05 & $0.71^{* * *}$ & \\
GY & $-0.72^{* * * *}$ & $-0.72^{* * * *}$ & $-0.89^{* * * *}$ & $0.45^{* * * *}$ & -0.06 & $0.14^{* *}$ & $0.87^{* * * *}$ & $0.71^{* * * *}$ \\
\hline
\end{tabular}

$\mathrm{CTa}, \mathrm{CTg} 1, \mathrm{CTg} 2$, canopy temperature during anthesis, mid- and late grain-filling stage, respectively; CCIa, CCIg1, $\mathrm{CCIg} 2$, chlorophyll content index during anthesis, mid- and late grain-filling stage, respectively; $\mathrm{PH}$, plant height; TKW, thousand kernels weight; GY, grain yield; ${ }^{*}, p<0.05 ; * *, p<0.01 ;{ }^{* * *}, p<0.001 ;{ }^{* * * *}, p<0.0001$.

\subsection{Marker Analysis and Construction of LGs}

Of 81,587 SNPs on the $90 \mathrm{~K}$ iSelect SNP array and 300 selected SSRs, only 9687 SNP marker and 44 SSRs were polymorphic between the parental lines and used for the construction of the linkage map. Both parental lines carried Ppd-D1a and Rht-D1b, but not Rht-B1b.

We identified $43 \mathrm{LGs}$ that represented all the 21 chromosomes of wheat (Table S2). Chr. 1A, 5A, 4B, 6B, and 7B were represented by one $\mathrm{LG}$ each; Chr. 2A, 4A, 6A, 1B, 3B, 5B, 1D, 2D, 3D, 4D, and 7D by two LGs each; Chr. 3A, 7A, 5D, and 6D by three LGs each; and Chr. 2B by four LGs (Table S2). The linkage map had a total length of $3633.19 \mathrm{~cm}$, whereas each LG had an average length of $173.01 \mathrm{~cm}$, ranging from $54.61 \mathrm{~cm}$ (Chr. 1D) to $261.51 \mathrm{~cm}$ (Chr. 5A). The average number of markers on each chromosome was 463.5, ranging from 15 on Chr. 3D to 1125 on Chr. 6A. Although markers on Chr. 1D, 3D, 4D, and 7D were low in number, they had a good distribution. The average marker density was $0.37 \mathrm{~cm}$, ranging from $0.19(\mathrm{Chr} .6 \mathrm{~A})$ to 5.56 (Chr. 3D). The map of A genome included 4320 markers (44.4\%) and had a total length of $1420.51 \mathrm{~cm}$ with an average marker density of $0.33 \mathrm{~cm}$; the map of B genome included 4367 markers $(44.9 \%)$ and had a total length of $1571.15 \mathrm{~cm}$ with an average marker density of $0.36 \mathrm{~cm}$; and the map of D genome included 1046 markers (10.7\%) and had a total length of $641.53 \mathrm{~cm}$ with an average marker density of $0.61 \mathrm{~cm}$. The $\mathrm{D}$ genome had the lowest marker coverage, suggesting that more markers were polymorphic in the A and B genomes. Of 9687 SNPs, only 357 were newly mapped (Table S3).

\subsection{QTL Detection for Physiological and Agronomic Traits}

After further optimization, 1002 SNP makers and 30 SSRs were used for QTL detection. We identified 116 QTL for all traits within and across environments; 71 QTL for CT and CCI, and 45 QTL for PH, TKW, and GY (Tables 4 and 5 and Table S4; Figure 1).

Table 4. Quantitative trait locus (QTL) detected for canopy temperature in the DH population of UI Platinum/SY Capstone.

\begin{tabular}{|c|c|c|c|c|c|c|c|c|c|}
\hline Traits & Trial & QTL $^{a}$ & Peak Marker & LG & Position $b$ & Marker Interval & LOD $^{c}$ & Add $^{d}$ & $\mathrm{R}^{2 \mathrm{e}}$ \\
\hline \multirow[t]{3}{*}{$\mathrm{CTa}$} & 15ABE1 & Q.CTa.ui-2A-2 & IWB21598 & $2 \mathrm{~A}-2$ & 11.05 & IWB3678-IWB21598 & 2.5 & 0.30 & 9.8 \\
\hline & & Q.CTa.ui-6B & IWB10604 & $6 \mathrm{~B}$ & 3.64 & IWB75959-IWB10604 & 5.8 & -0.48 & 21.6 \\
\hline & 15ABE2 & Q.CTa.ui-2B-2.1 & IWB21394 & $2 \mathrm{~B}-2$ & 8.2 & IWB21394-IWB67534 & 5.5 & 0.77 & 20.4 \\
\hline
\end{tabular}


Table 4. Cont.

\begin{tabular}{|c|c|c|c|c|c|c|c|c|c|}
\hline Traits & Trial & QTL $^{a}$ & Peak Marker & LG & Position $^{b}$ & Marker Interval & LOD $^{c}$ & Add $^{d}$ & $R^{2 e}$ \\
\hline & \multirow{6}{*}{ 15ABE3 } & Q.CTa.ui-2B-2.2 & IWB594 & 2B-2 & 44.63 & IWB11092-IWB594 & 2.5 & -0.51 & 10.0 \\
\hline & & Q.CTa.ui-2B-3 & IWB35482 & 2B-3 & 63.36 & IWB35482-IWB74377 & 2.7 & 0.40 & 10.3 \\
\hline & & Q.CTa.ui-4B.1 & IWB10190 & $4 B$ & 29.65 & IWB20226-IWB57507 & 4.0 & -0.48 & 15.5 \\
\hline & & Q.CTa.ui-5B-2 & IWB626 & 5B-2 & 42.9 & IWB36579-IWB18101 & 5.8 & -0.62 & 21.7 \\
\hline & & Q.CTa.ui-2B-3 & IWB35482 & 2B-3 & 55.36 & IWB35482-IWB74377 & 3.0 & 0.29 & 11.6 \\
\hline & & Q.CTa.ui-4B.1 & IWB10190 & $4 B$ & 29.65 & IWB20226-IWB57507 & 3.3 & -0.44 & 12.8 \\
\hline & \multirow[t]{3}{*}{ 16ABE4 } & Q.CTa.ui-3B-1 & IWB10973 & 3B-1 & 82.18 & IWB21837-IWB10973 & 2.6 & 0.62 & 10.4 \\
\hline & & Q.CTa.ui-4B.1 & IWB10190 & $4 B$ & 29.65 & IWB20226-IWB57507 & 2.6 & -0.41 & 11.2 \\
\hline & & Q.CTa.ui-4B.2 & IWB59992 & $4 \mathrm{~B}$ & 84.48 & IWB27326-IWB21502 & 2.8 & -0.64 & 11.0 \\
\hline & \multirow[t]{2}{*}{ Average ${ }^{f}$} & Q.CTa.ui-1A & IWB36144 & $1 \mathrm{~A}$ & 66.72 & IWB21167-IWB36144 & 2.5 & 0.23 & 10.1 \\
\hline & & Q.CTa.ui-4B.1 & IWB10190 & $4 B$ & 29.65 & IWB20226-IWB57507 & 3.8 & -0.47 & 14.6 \\
\hline \multirow[t]{15}{*}{ CTg1 } & \multirow[t]{2}{*}{ 15ABE1 } & Q.CTg1.ui-5A.2 & IWB65470 & $5 \mathrm{~A}$ & 14.26 & IWB65470-IWB13571 & 3.4 & -1.47 & 13.3 \\
\hline & & Q.CTg1.ui-3D-1 & IWB63148 & 3D-1 & 45.53 & IWB63148 -IWB51532 & 2.8 & 1.18 & 11.3 \\
\hline & \multirow[t]{4}{*}{ 15ABE2 } & Q.CTg1.ui-6A-2 & IWB12439 & $6 \mathrm{~A}-2$ & 88.2 & IWB12439-IWB13795 & 2.5 & 0.20 & 9.8 \\
\hline & & Q.CTg1.ui-2B-4 & Barc13 & 2B-4 & 26 & Barc13-IWB22058 & 2.8 & 0.26 & 11.1 \\
\hline & & Q.CTg1.ui-5B-2.1 & IWB80334 & $5 B-2$ & 10.96 & IWB80334-IWB29709 & 3.1 & -0.24 & 12.3 \\
\hline & & Q.CTg1.ui-5B-2.2 & IWB19921 & $5 \mathrm{~B}-2$ & 20.97 & IWB36033-IWB19921 & 3.3 & -0.24 & 12.8 \\
\hline & \multirow[t]{4}{*}{ 15ABE3 } & Q.CTg1.ui-7A-3 & IWB49784 & $7 A-3$ & 52.99 & IWB25307-IWB34223 & 4.7 & 0.41 & 17.8 \\
\hline & & Q.CTg1.ui-1B-1 & IWB58775 & 1B-1 & 128 & IWB10780-IWB58775 & 2.9 & -0.31 & 13.6 \\
\hline & & Q.CTg1.ui-2B-3 & IWB18944 & $2 B-3$ & 22 & IWB18944-Wmc149 & 2.6 & -0.34 & 10.2 \\
\hline & & Q.CTg1.ui-5B-2.1 & IWB80334 & $5 B-2$ & 10.96 & IWB80334-IWB29709 & 2.8 & -0.23 & 11.1 \\
\hline & \multirow{2}{*}{ 16ABE4 } & Q.CTg1.ui-5B-2.1 & IWB80334 & $5 B-2$ & 10.96 & IWB80334-IWB29709 & 2.6 & -0.35 & 10.4 \\
\hline & & Q.CTg1.ui-6B & IWB10604 & $6 \mathrm{~B}$ & 3.64 & IWB75959-IWB10604 & 2.9 & -0.67 & 11.5 \\
\hline & \multirow[t]{3}{*}{ Average ${ }^{f}$} & Q.CTg1.ui-3B-1 & IWB8756 & 3B-1 & 1.82 & IWB11545-IWB63436 & 3.8 & -0.37 & 14.8 \\
\hline & & Q.CTg1.ui-5B-2.1 & IWB80334 & $5 B-2$ & 10.96 & IWB80334-IWB29709 & 2.6 & -0.35 & 10.2 \\
\hline & & Q.CTg1.ui-5B-2.3 & IWB36579 & $5 \mathrm{~B}-2$ & 41.08 & IWB36579-IWB18101 & 4.2 & 0.49 & 16.2 \\
\hline \multirow[t]{15}{*}{$\mathrm{CTg} 2$} & \multirow{3}{*}{ 15ABE1 } & Q.CTg2.ui-1B-1 & IWB58775 & 1B-1 & 128 & IWB10780-IWB58775 & 3.5 & 6.69 & 13.6 \\
\hline & & Q.CTg2.ui-6B.1 & IWB10400 & $6 B$ & 88.11 & IWB65137-IWB10400 & 3.8 & 2.32 & 14.5 \\
\hline & & Q.CTg2.ui-5D-1 & IWB79949 & 5D-1 & 0 & IWB79949-IWB4139 & 3.7 & -2.28 & 14.5 \\
\hline & \multirow[t]{3}{*}{ 15ABE2 } & Q.CTg2.ui-2B-2 & Barc18 & 2B-2 & 21.88 & IWB22675-IWB11319 & 3.9 & 0.30 & 15.1 \\
\hline & & Q.CTg2.ui-4B.1 & IWB10190 & $4 \mathrm{~B}$ & 29.65 & IWB20226-IWB57507 & 3.2 & -0.27 & 12.4 \\
\hline & & Q.CTg2.ui-5B-2 & IWB29709 & $5 B-2$ & 13.69 & IWB80334-IWB29709 & 3.3 & -0.29 & 13.0 \\
\hline & \multirow[t]{3}{*}{ 15ABE3 } & Q.CTg2.ui-3A-1 & IWB33546 & $3 \mathrm{~A}-1$ & 2 & IWB33546-IWB14315 & 2.5 & 0.26 & 9.8 \\
\hline & & Q.CTg2.ui-6B.1 & IWB10400 & $6 B$ & 88.11 & IWB65137-IWB10400 & 2.9 & 1.47 & 10.2 \\
\hline & & Q.CTg2.ui-2D-1 & cfd73 & 2D-1 & 108.19 & cfd73-IWB1093 & 3.1 & 0.29 & 12.1 \\
\hline & \multirow[t]{2}{*}{ 16ABE4 } & Q.CTg2.ui-6B.1 & IWB10400 & $6 B$ & 88.11 & IWB65137-IWB10400 & 2.6 & 1.08 & 10.4 \\
\hline & & Q.CTg2.ui-6B.2 & IWB75959 & $6 \mathrm{~B}$ & 0 & IWB75959-IWB10604 & 5.2 & -0.76 & 19.7 \\
\hline & \multirow[t]{4}{*}{ Average $^{f}$} & Q.CTg2.ui-1A & IWB36377 & $1 \mathrm{~A}$ & 33.94 & IWB36377-IWB21518 & 4.0 & -0.61 & 15.3 \\
\hline & & Q.CTg2.ui-7A-1 & IWB46359 & 7A-1 & 48.95 & IWB25077-IWB46359 & 2.6 & -0.49 & 10.3 \\
\hline & & Q.CTg2.ui-6B.1 & IWB10400 & $6 B$ & 88.11 & IWB65137-IWB10400 & 2.6 & 0.47 & 10.3 \\
\hline & & Q.CTg2.ui-2D-2 & IWB11455 & $2 \mathrm{D}-2$ & 20.15 & IWB12523-IWB11455 & 2.8 & -0.52 & 11.1 \\
\hline
\end{tabular}

15ABE1, 15ABE2, 15ABE3: trial 1, 2 and 3 in Aberdeen in 2015, respectively; 16ABE4, trial 4 in Aberdeen in 2016. ${ }^{a} \mathrm{CTa}$, CTg1, CTg2, canopy temperature during anthesis, mid- and late grain-filling stage, respectively; ui, university of Idaho. Different QTL on the same linkage group was indicated with 1 and 2 after the period following the linkage group. ${ }^{b}$ Position of QTL located on linkage group, as CM distance from the top of each linkage group. ${ }^{c}$ An LOD threshold of 2.5 was used for declaration of QTL. ${ }^{\mathrm{d}}$ Positive "additive effect" indicated effect from SY Capstone, negative "additive effect" indicated effect from UI Platinum. e Percentage of phenotypic variation explained by the QTL. ${ }^{\mathrm{f}}$ Average data across the four environments was used. LG, linkage group. Italic and bold showed the stable QTL for traits.

For CTa, 10 QTL were detected on Chr. 1A, 2A-2, 2B-2, 2B-3, 3B-1, 4B, 5B-2, and 6B, explaining 9.8-21.7\% of the phenotypic variation across environments (Tables 4 and S4; Figure 1). Two QTL on Chr. 2B-3 between IWB35482 and IWB74377 at 55.36-63.62 cm and on Chr. 4B between IWB20226 and IWB57507 at 27.83-30.56 cm were found in two (15ABE2 and 15ABE3) and four environments (15ABE2, 15ABE3, 16ABE4 and Average environment), respectively, explaining $10.3 \%-11.6 \%$ and $11.2 \%-14.6 \%$ of the phenotypic variation, respectively.

For CTg1, 12 QTL were detected on Chr. 5A, 6A-2, 7A-3, 1B-1, 2B-3, 2B-4, 3B-1, 5B-2, 6B, and 3D-1, explaining $9.8 \%-17.8 \%$ of the phenotypic variation across environments (Tables 4 and S4; Figure 1). A stable QTL on Chr. 5B-2 tightly linked to IWB80334 at 10.96-13.69 cm was found in four environments (15ABE2, $15 \mathrm{ABE} 3,16 \mathrm{ABE} 4$ and Average environment), explaining $10.2 \%-12.3 \%$ of the phenotypic variation.

For CTg2, 12 QTL were detected on Chr. 1A, 3A-1, 7A-1, 1B-1, 2B-2, 4B, 5B-2, 6B, 2D-1, 2D-2, and 5D-1, explaining $9.8 \%-19.7 \%$ of the phenotypic variation across environments (Tables 4 and S4; Figure 1). A QTL on Chr. 6B between IWB65137 and IWB10400 at 87.2-88.11 cm was stable in four environments (15ABE1, $15 \mathrm{ABE} 3,16 \mathrm{ABE} 4$ and Average environment), explaining $10.2 \%-14.5 \%$ of the phenotypic variation.

For CCIa, 15 QTL were detected on the homologous chromosome groups 2 and 6 as well as on Chr. 3A-1, 3A-2, 4A-1, 5A, 7A-2, 7B, 3D-1, 3D-2, and 4D-1, explaining 9.9\%-19.2\% of the phenotypic 
variation across environments (Tables 5 and S4; Figure 1). Three QTL on Chr. 3A-2, 7A-2, and 3D-2 were found in two environments, each explaining $11.6 \%-19.1 \%$ of the phenotypic variation.

For CCIg1, nine QTL were detected on Chr. 3A-2, 4A-1, 5A, 1D-1, 1D-2, 2D-1, and 2B-2, explaining $11.0 \%-31.3 \%$ of the phenotypic variation across environments (Tables 5 and S4; Figure 1). A QTL on Chr. 5A between IWB14149 and IWB10765 at 91.35-93.17 cm was found in four environments (15ABE2, 15ABE3, 16ABE4 and Average environment), explaining $15.2 \%-30.6 \%$ of the phenotypic variation. Additionally, two QTL on Chr. 3A-2 between IWB17683 and IWB13444 at 51.17-52.08 cm and on Chr. 2D-1 between IWB15442 and IWB60397 at 133.01-134.83 cm were found in two environments, explaining $11.9 \%-12.2 \%$ and $11.8 \%-20.6 \%$ of the phenotypic variation, respectively.

Table 5. QTL detected for chlorophyll content index in the DH population of UI Platinum/SY Capstone.

\begin{tabular}{|c|c|c|c|c|c|c|c|c|c|}
\hline Traits & Trial & QTL $^{a}$ & Peak Marker & LG & Position $b$ & Marker Interval & LOD $^{c}$ & Add ${ }^{d}$ & $R^{2} \mathrm{e}$ \\
\hline \multirow[t]{18}{*}{ CCIa } & 15ABE1 & Q.CCIa.ui-3D-1 & IWB45650 & $3 \mathrm{D}-1$ & 18 & IWB45650-Wms3 & 2.6 & -3.44 & 10.3 \\
\hline & \multirow[t]{5}{*}{ 15ABE2 } & Q.CCIa.ui-3A-2 & IWB10063 & $3 \mathrm{~A}-2$ & 87.62 & IWB11450-IWB10063 & 3.9 & 2.99 & 15.1 \\
\hline & & Q.CCIa.ui-7B & IWB6754 & $7 \mathrm{~B}$ & 233.72 & IWB25433-IWB6754 & 4.1 & -3.23 & 15.9 \\
\hline & & Q.CCIa.ui-3D-2 & IWB9674 & $3 \mathrm{D}-2$ & 16.91 & IWB9674-IWB71478 & 3.0 & 3.23 & 11.9 \\
\hline & & Q.CCIa.ui-4D-1 & IWB8050 & $4 \mathrm{D}-1$ & 45.41 & IWB14984-IWB8050 & 2.9 & -2.60 & 11.4 \\
\hline & & Q.CCIa.ui-6D-3 & IWB13767 & $6 \mathrm{D}-3$ & 0.91 & IWB13767-IWB46507 & 2.5 & 2.42 & 10.0 \\
\hline & \multirow[t]{3}{*}{ 15ABE3 } & Q.CCIa.ui-4A-1 & IWB12946 & $4 \mathrm{~A}-1$ & 78.95 & IWB37346-IWB10035 & 3.6 & 1.89 & 13.9 \\
\hline & & Q.CCIa.ui-7A-2 & IWB12020 & $7 \mathrm{~A}-2$ & 81.58 & IWB39758-IWB12020 & 4.1 & -1.45 & 15.9 \\
\hline & & Q.CCIa.ui-2D-1 & IWB15442 & 2D-1 & 133.01 & IWB15442-IWB60397 & 3.4 & 1.86 & 13.4 \\
\hline & \multirow[t]{4}{*}{ 16ABE4 } & Q.CCIa.ui-2A-1 & IWB10499 & $2 \mathrm{~A}-1$ & 12.87 & IWB10836-IWB29812 & 3.2 & 3.26 & 12.5 \\
\hline & & Q.CCIa.ui-3A-1 & IWB33546 & $3 \mathrm{~A}-1$ & 2 & IWB33546-IWB14315 & 2.8 & -2.20 & 11.2 \\
\hline & & Q.CCIa.ui-5A & IWB21197 & $5 \mathrm{~A}$ & 165.38 & IWB21197-IWB35869 & 2.9 & 2.16 & 11.4 \\
\hline & & Q.CCIa.ui-6A-2 & IWB19789 & $6 \mathrm{~A}-2$ & 70.82 & IWB22680-IWB19789 & 3.3 & -2.31 & 13.0 \\
\hline & \multirow[t]{5}{*}{ Average $\mathrm{f}^{\mathrm{f}}$} & Q.CCIa.ui-3A-2 & IWB10063 & $3 \mathrm{~A}-2$ & 87.62 & IWB11450-IWB10063 & 2.9 & 1.20 & 11.6 \\
\hline & & Q.CCIa.ui-7A-2 & IWB12020 & $7 \mathrm{~A}-2$ & 81.58 & IWB39758-IWB12020 & 5.1 & -1.71 & 19.1 \\
\hline & & Q.CCIa.ui-2B-3 & IWB18944 & $2 \mathrm{~B}-3$ & 22 & IWB18944-Wmc149 & 2.5 & -1.28 & 9.9 \\
\hline & & Q.CCIa.ui-6B & IWB58083 & $6 \mathrm{~B}$ & 68.05 & IWB52064-IWB58083 & 5.1 & 1.66 & 19.2 \\
\hline & & Q.CCIa.ui-3D-2 & IWB9674 & $3 \mathrm{D}-2$ & 4.91 & IWB9674-IWB71478 & 3.9 & 1.54 & 15.1 \\
\hline \multirow[t]{15}{*}{ CCIg1 } & \multirow[t]{3}{*}{ 15ABE1 } & Q.CCIg1.ui-2B-2.1 & IWB67534 & $2 \mathrm{~B}-2$ & 10.93 & IWB67534-IWB13631 & 4.2 & 9.26 & 16.3 \\
\hline & & Q.CCIg1.ui-2B-2.2 & Barc18 & $2 \mathrm{~B}-2$ & 21.88 & IWB22675-IWB11319 & 3.2 & -6.80 & 12.5 \\
\hline & & Q.CCIg1.ui-2D-1 & IWB15442 & $2 \mathrm{D}-1$ & 133.01 & IWB15442-IWB60397 & 3.0 & 3.68 & 11.8 \\
\hline & \multirow[t]{3}{*}{ 15ABE2 } & Q.CCIg1.ui-4A-1 & IWB12946 & $4 \mathrm{~A}-1$ & 78.95 & IWB37346-IWB10035 & 2.8 & 2.73 & 11.1 \\
\hline & & Q.CCIg1.ui-5A.1 & IWB14149 & $5 A$ & 91.35 & IWB14149-IWB10765 & 3.9 & 3.09 & 15.2 \\
\hline & & Q.CCIg1.ui-5A.2 & IWB35869 & $5 \mathrm{~A}$ & 166.29 & IWB21197-IWB17983 & 9.0 & -5.45 & 31.3 \\
\hline & \multirow[t]{3}{*}{ 15ABE3 } & Q.CCIg1.ui-3A-2 & IWB17683 & $3 \mathrm{~A}-2$ & 51.17 & IWB17683-IWB13444 & 3.1 & -2.70 & 12.2 \\
\hline & & Q.CCIg1.ui-5A.1 & IWB14149 & $5 A$ & 91.35 & IWB14149-IWB10765 & 4.7 & 3.36 & 17.8 \\
\hline & & Q.CCIg1.ui-2D-1 & IWB15442 & 2D-1 & 133.01 & IWB15442-IWB60397 & 4.7 & 3.02 & 18.0 \\
\hline & \multirow[t]{3}{*}{ 16ABE4 } & Q.CCIg1.ui-3A-2 & IWB17683 & $3 \mathrm{~A}-2$ & 51.17 & IWB17683-IWB13444 & 3.0 & -2.20 & 11.9 \\
\hline & & Q.CCIg1.ui-5A.1 & IWB14149 & $5 A$ & 91.35 & IWB14149-IWB10765 & 8.7 & 5.21 & 30.6 \\
\hline & & Q.CCIg1.ui-1D-2 & IWB65713 & $1 \mathrm{D}-2$ & 0 & IWB65713-IWB55046 & 3.6 & 2.42 & 14.1 \\
\hline & \multirow[t]{3}{*}{ Average ${ }^{f}$} & Q.CCIg1.ui-5A.1 & IWB14149 & $5 A$ & 91.35 & IWB14149-IWB10765 & 5.9 & 5.34 & 21.8 \\
\hline & & Q.CCIg1.ui-1D-1 & IWB42629 & 1D-1 & 19.94 & IWB42629-IWB80211 & 2.8 & -1.64 & 11.0 \\
\hline & & Q.CCIg1.ui-2D-1 & IWB15442 & $2 \mathrm{D}-1$ & 133.01 & IWB15442-IWB60397 & 5.5 & 2.22 & 20.6 \\
\hline \multirow[t]{16}{*}{ CCIg2 } & \multirow[t]{2}{*}{ 15ABE1 } & Q.CCIg2.ui-1B-1 & IWB58775 & 1B-1 & 128 & IWB10780-IWB58775 & 3.0 & 3.08 & 11.7 \\
\hline & & Q.CCIg2.ui-6D-3 & IWB46507 & $6 \mathrm{D}-3$ & 0 & IWB46507-IWB13767 & 2.9 & -3.07 & 11.3 \\
\hline & \multirow[t]{5}{*}{ 15ABE2 } & Q.CCIg2.ui-4A-1 & IWB12946 & $4 \mathrm{~A}-1$ & 78.95 & IWB37346-IWB10035 & 3.0 & 2.74 & 11.7 \\
\hline & & Q.CCIg2.ui-5A.1 & IWB11865 & $5 \mathrm{~A}$ & 167.2 & IWB21197-IWB17983 & 6.6 & -4.33 & 24.1 \\
\hline & & Q.CCIg2.ui-1B-2.1 & IWB27496 & $1 \mathrm{~B}-2$ & 38.81 & IWB5753-IWB27496 & 3.1 & 4.99 & 12.2 \\
\hline & & Q.CCIg2.ui-1B-2.2 & IWB21945 & $1 \mathrm{~B}-2$ & 53.38 & IWB21945-IWB14060 & 2.7 & -4.33 & 10.7 \\
\hline & & Q.CCIg2.ui-3B-1 & IWB5790 & 3B-1 & 50.52 & IWB5790-IWB10800 & 3.5 & -3.12 & 13.7 \\
\hline & \multirow[t]{3}{*}{ 15ABE3 } & Q.CCIg2.ui-1A & IWB12695 & $1 \mathrm{~A}$ & 157.15 & IWB11395-IWB13624 & 6.6 & 2.93 & 24.1 \\
\hline & & Q.CCIg2.ui-5B-2 & IWB5882 & $5 \mathrm{~B}-2$ & 43.81 & IWB36579-IWB18101 & 3.7 & 2.17 & 14.3 \\
\hline & & Q.CCIg2.ui-2D-1 & IWB15442 & 2D-1 & 133.01 & IWB15442-IWB60397 & 6.9 & 2.98 & 25.2 \\
\hline & \multirow[t]{4}{*}{ 16ABE4 } & Q.CCIg2.ui-3A-2 & IWB29612 & $3 \mathrm{~A}-2$ & 100.63 & IWB29612-IWB63761 & 2.6 & -2.14 & 10.2 \\
\hline & & Q.CCIg2.ui-5A.1 & IWB11865 & $5 \mathrm{~A}$ & 167.2 & IWB21197-IWB17983 & 3.9 & -3.73 & 14.9 \\
\hline & & Q.CCIg2.ui-5A.2 & IWB14149 & $5 \mathrm{~A}$ & 91.35 & IWB14149-IWB10765 & 6.2 & 4.58 & 22.7 \\
\hline & & Q.CCIg2.ui-7A-3 & IWB27037 & $7 \mathrm{~A}-3$ & 0 & IWB27037-IWB65210 & 4.0 & -2.70 & 15.5 \\
\hline & \multirow[t]{2}{*}{ Average $\mathrm{f}^{\mathrm{f}}$} & Q.CCIg2.ui-1A & IWB13624 & $1 \mathrm{~A}$ & 158.79 & IWB44568-IWB13624 & 2.7 & 1.38 & 10.6 \\
\hline & & Q.CCIg2.ui-3B-1 & IWB5790 & $3 \mathrm{~B}-1$ & 50.52 & IWB5790-IWB10800 & 3.8 & -1.59 & 14.6 \\
\hline
\end{tabular}

15ABE1, 15ABE2, 15ABE3: trial 1, 2 and 3 in Aberdeen in 2015, respectively; 16ABE4, trial 4 in Aberdeen in 2016. ${ }^{\text {a CCIa, }}$ CCIg1, CCIg2, chlorophyll content index during anthesis, mid- and late grain-filling stage, respectively; ui, university of Idaho. Different QTL on the same linkage group was indicated with 1 and 2 after the period following the linkage group.

${ }^{b}$ Position of QTL located on linkage group, as CM distance from the top of each linkage group. ${ }^{c}$ A LOD threshold of 2.5 was used for declaration of QTL. ${ }^{\mathrm{d}}$ Positive "additive effect" indicated effect from SY Capstone, negative "additive effect" indicated effect from UI Platinum. ${ }^{e}$ Percentage of phenotypic variation explained by the QTL. ${ }^{\mathrm{f}}$ Average data across the four environments was used. LG, linkage group. Italic and bold showed the stable QTL for traits. 

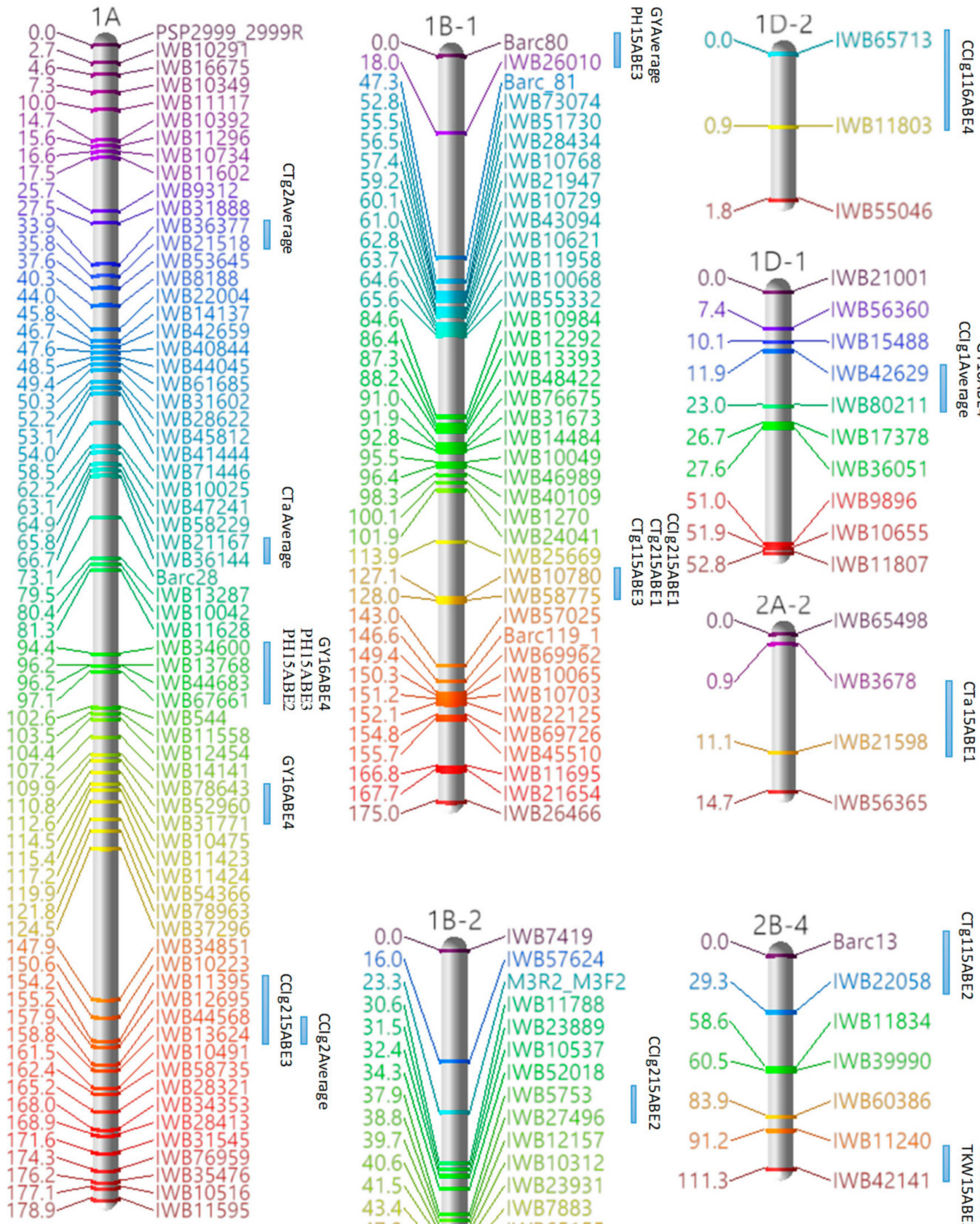

62.0

63.7) - IWB11958

64.6) - IN IWB10068

65.6 y IWB55332

86.4 /WWB12292

87.3 IWB13393

88.2) IWB48422

91.0 II IWB76675

$92.9-1$ WB

$92.8-1 W$
$95.5-1 W B 10049$

96.4 a N IWB46989

98.3 IWB40109

100.1 IWB1270

101.9 IWB24041

127.1 - WWB10780

128.0 - - IWB58775

143.0 146.6 C Carc1191

149.4 JWB69962

150.3 = IWB10065

$151.2=-$ IWB10703

152.12 WWB22125

154.8 NWB69726

155.7 IWB45510

$166.8-W B 11695$

IWB21654

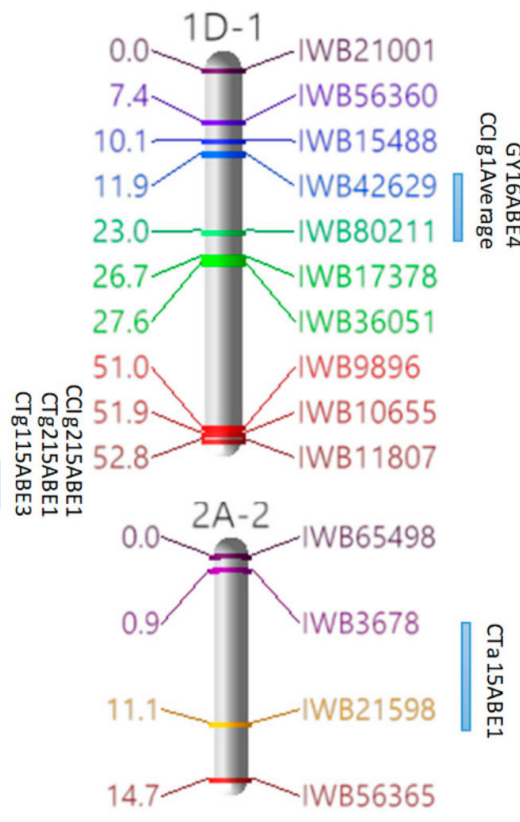

$1 \mathrm{~B}-2$

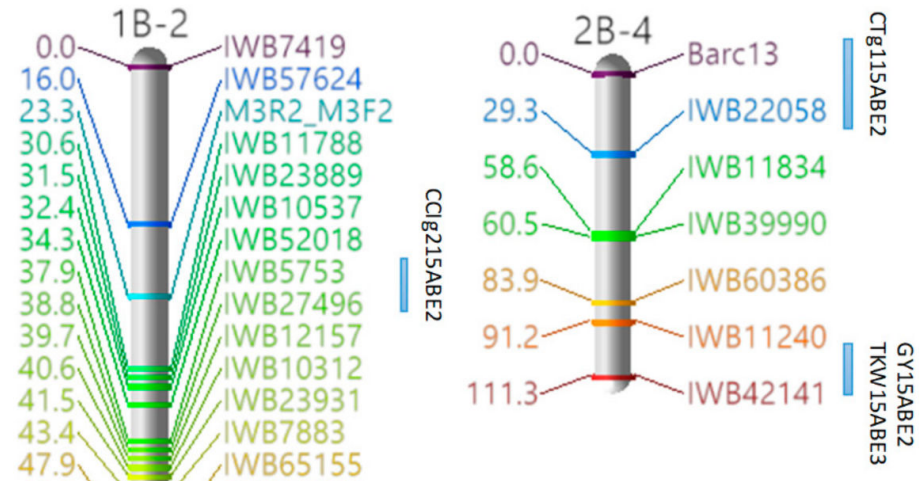

47.9 IE $=$ IWB65155

48.8 = IWB25756

49.7 IWB2106

52.5 IWB28998

$53.4-二=$ IWB21945

$55.2 \sim$ IWB14060

$57.0=$ Barc152

59.8 IWB30286

60.7 IWB11671

|

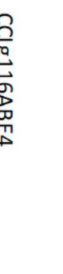

- IWB10908

$$
\text { 哭 }
$$

Figure 1. Cont. 

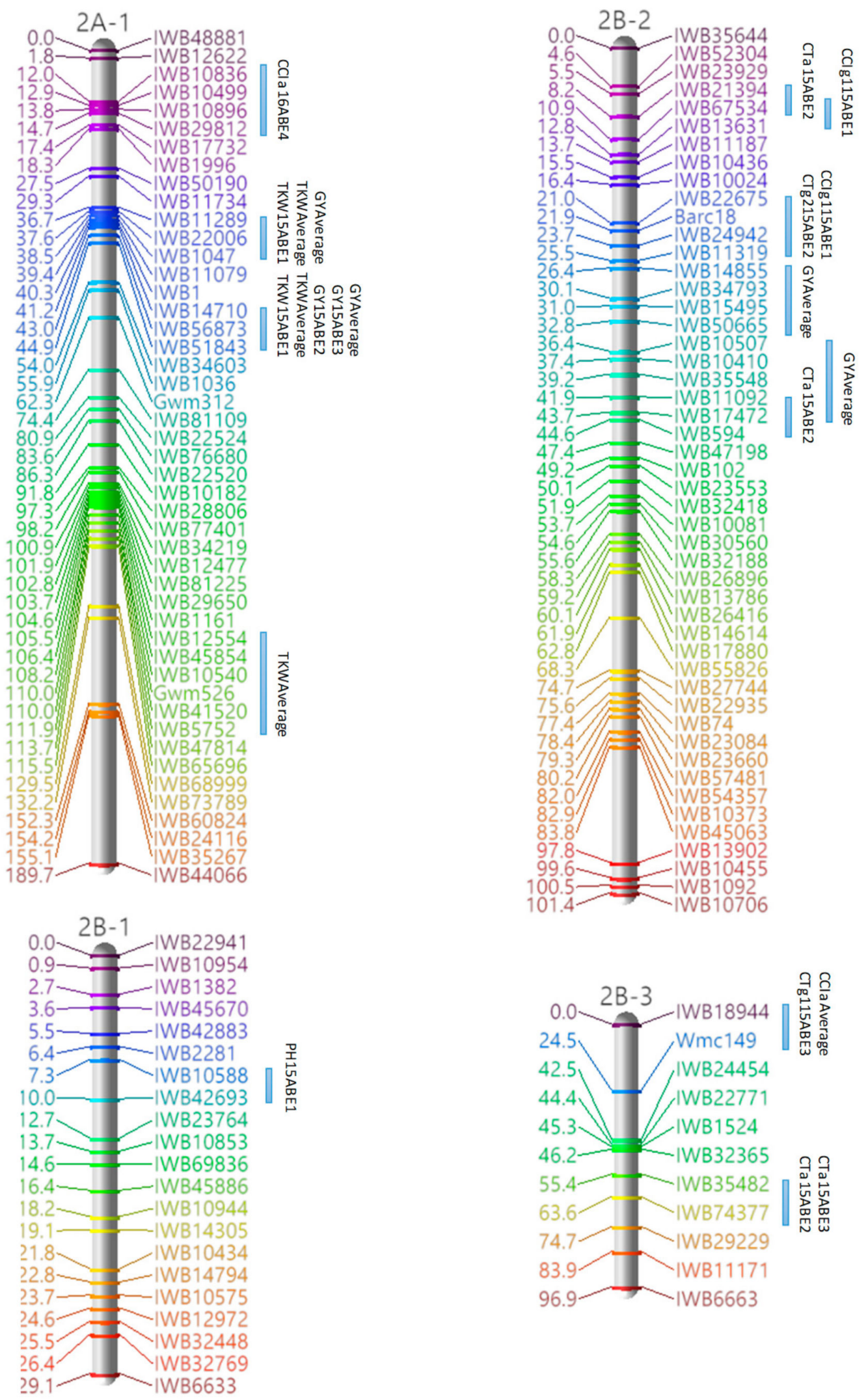

Figure 1. Cont. 

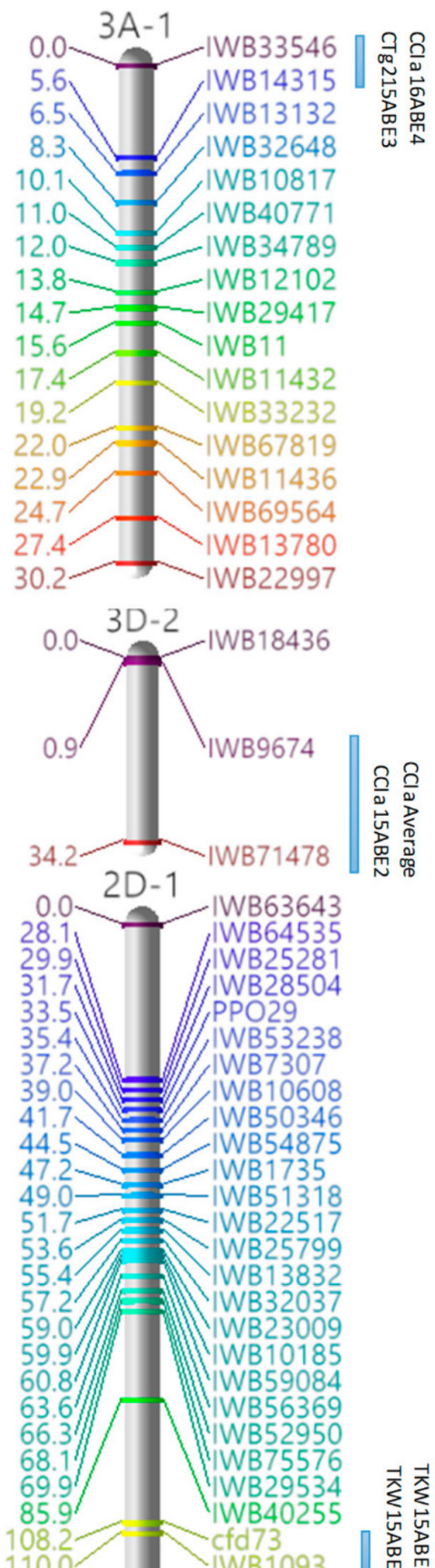

108.2
110.0

122.1—_W - IWB78851

$124.8-1 W B 38687$

$125.7 \%$ IWB24133

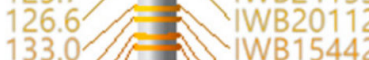

$\begin{aligned} & 133.0 \\ & 134.8\end{aligned} /$ = IWB15442

137.6 IWB

139.4 UWB19009

142.1 UWB39419

$155.1 \%$ IWB 11266

164.3

175.4

Gwm484

IWB75962

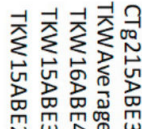

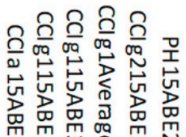

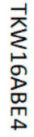
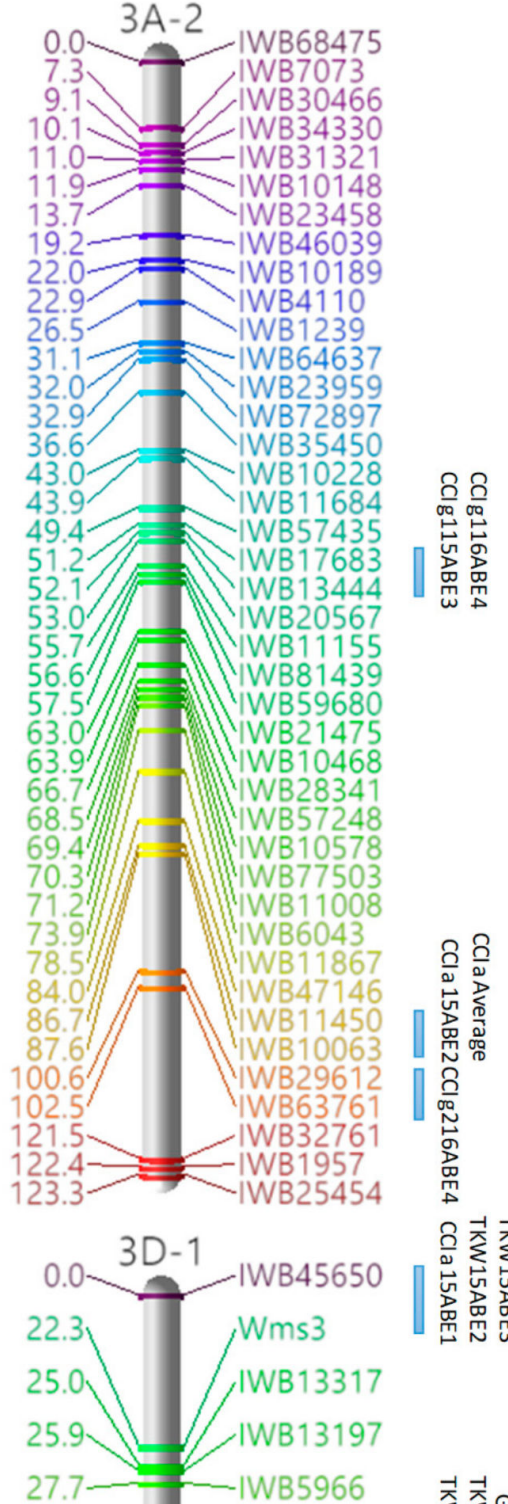

ถ츷츷

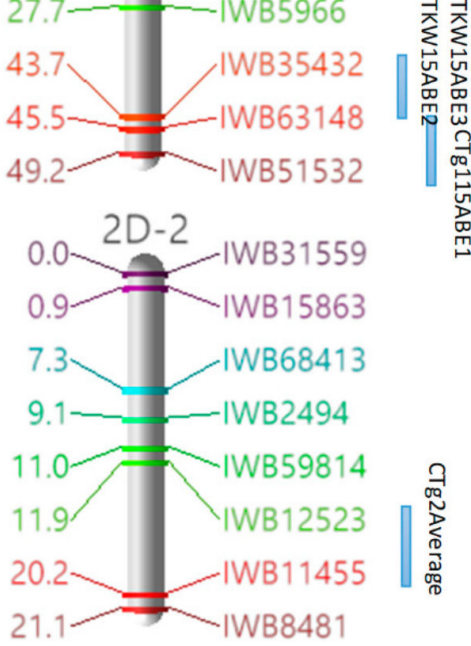

Figure 1. Cont. 

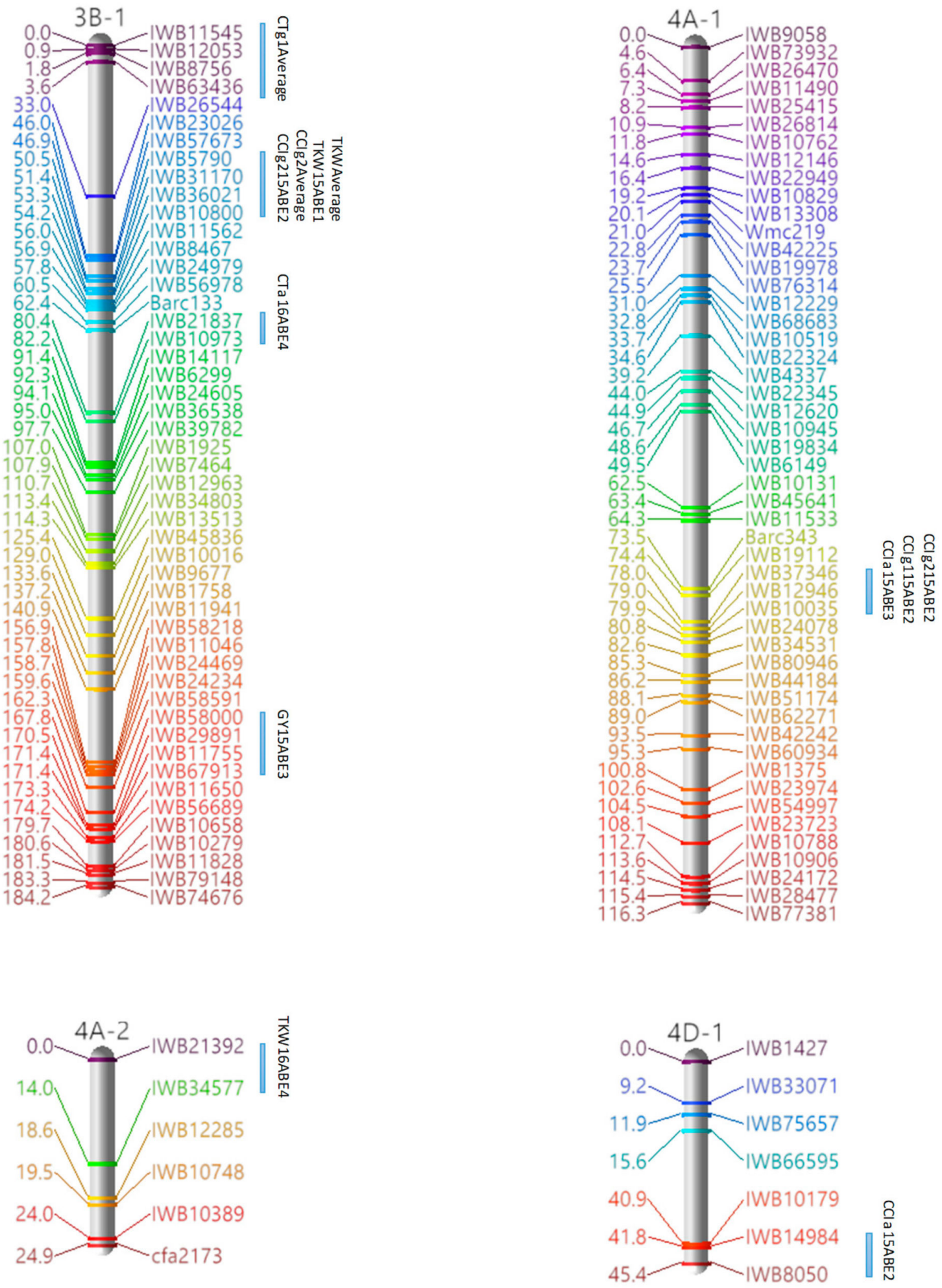

Figure 1. Cont. 

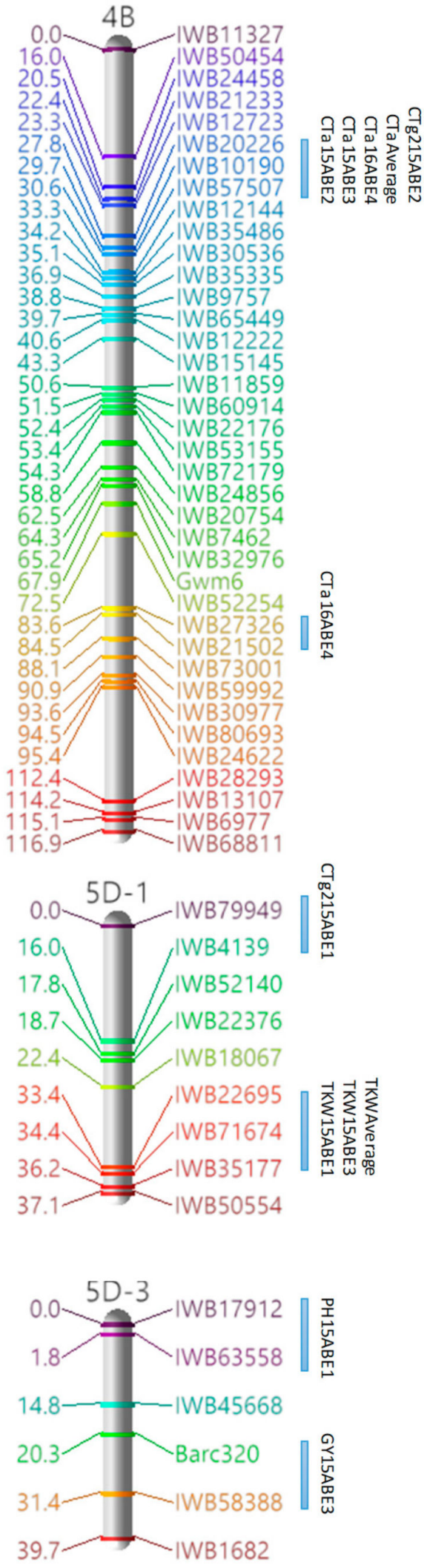

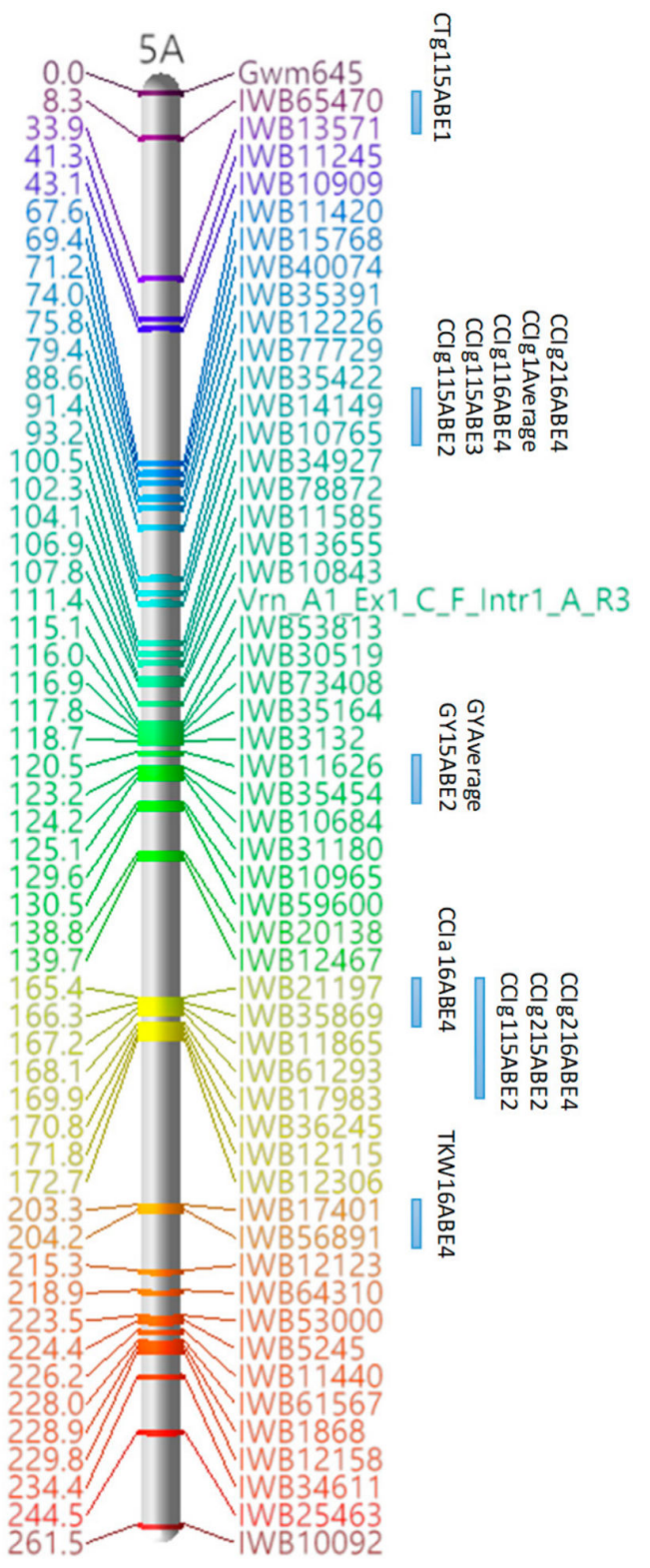

Figure 1. Cont. 

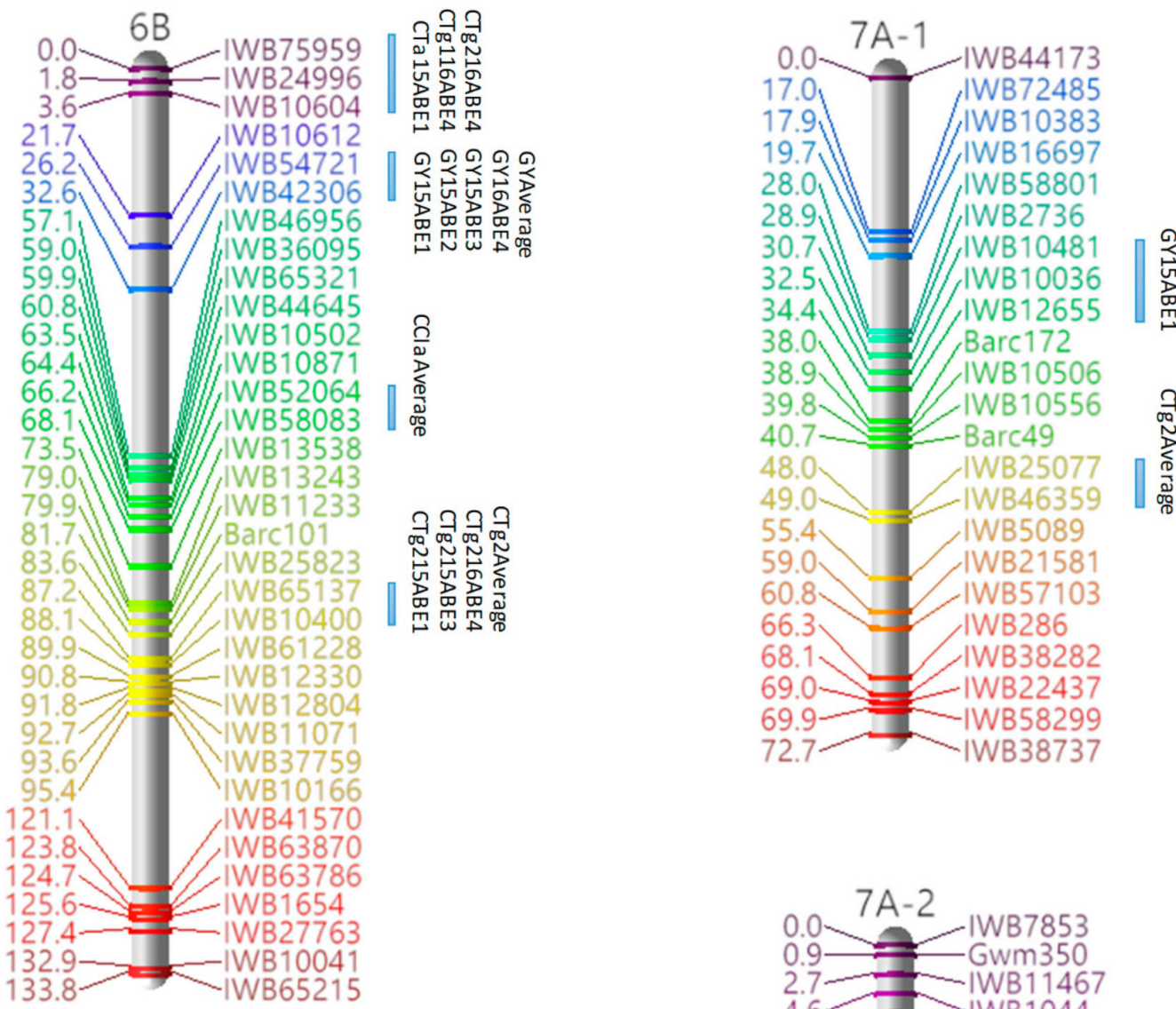

38.9 1 IWB10506

39.8 IWB10556

40.7 - - Barc49

48.0 -IWB25077

49.0- IWB46359

$55.4=\mid W B 5089$

59.0 IWB21581

60.8 IWB57103

$66.3=\quad$ IWB286

68.1 -IWB38282

$69.0=-1$ IWB22437

$69.9-$ IWB58299

$72.7 \longrightarrow$ IWB38737
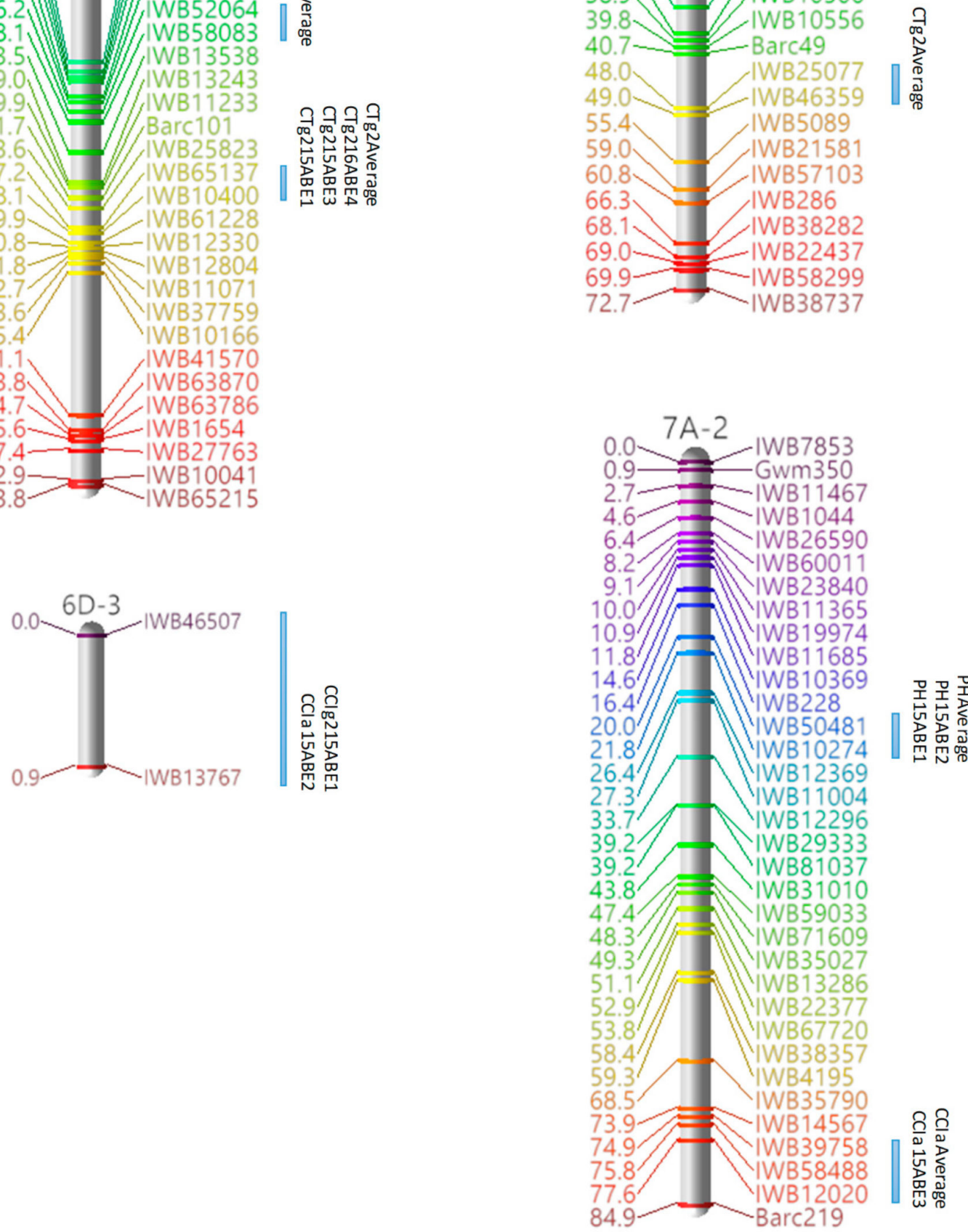

Figure 1. Cont. 

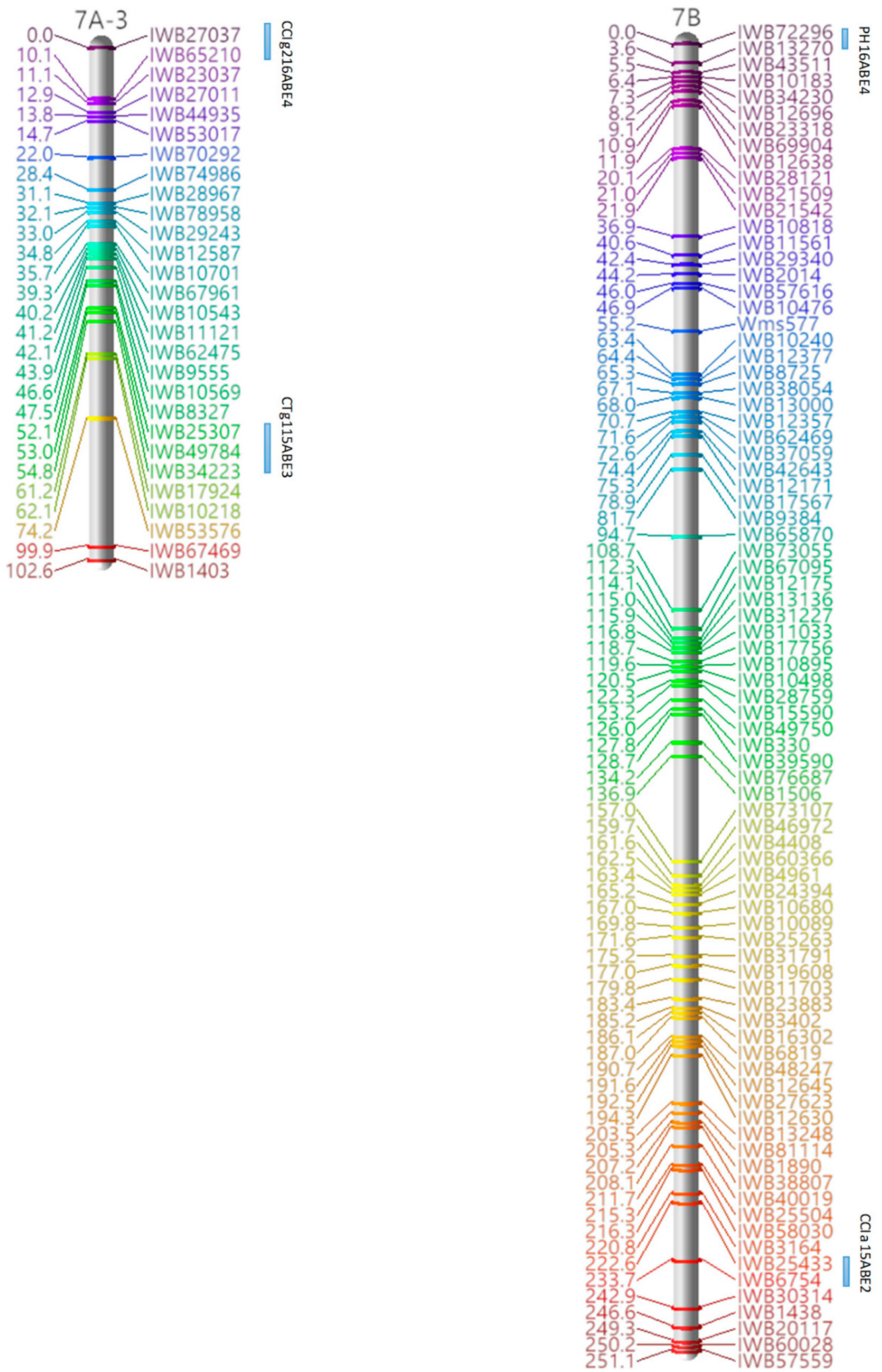

Figure 1. Quantitative trait locus (QTL) for physiological traits (CTa, CTg1, CTg2, CCI, , CCIg1 and CCIg2), $\mathrm{PH}, \mathrm{TKW}$ and GY on the linkage groups of the doubled haploid (DH) population from UI Platinum/SY Capstone. 15ABE1, 15ABE2, 15ABE3: trial 1, 2 and 3 in Aberdeen in 2015, respectively; 16ABE4, trial 4 in Aberdeen in 2016; CTa, CTg1, CTg2, canopy temperature during anthesis, mid-grain-filling and late grain-filling stage, respectively; CCIa, CCIg1, CCIg2, chlorophyll content index during anthesis, mid-grain-filling and late grain-filling stage, respectively; $\mathrm{PH}$, plant height; TKW, thousand kernels weight; GY, grain yield. 
For CCIg2, 13 QTL were detected on Chr. 1A, 3A-2, 4A-1, 5A, 7A-3, 1B-1, 1B-2, 3B-1, 5B-2, 2D-1, and $6 \mathrm{D}-3$, explaining $10.2 \%-25.2 \%$ of the phenotypic variation across environments (Tables 5 and S4; Figure 1). A QTL on Chr. 5A between IWB21197 and IWB17983 at $165.38-169.93 \mathrm{~cm}$ was found in two environments (15ABE2 and 15ABE4), explaining $14.9 \%-24.1 \%$ of the phenotypic variation.

For PH, 12 QTL were detected on Chr. 1A, 5A, 6A-2, 7A-2, 1B-1, 2B-1, 5B-2, 7B, 2D-1, and 5D-3, explaining 9.9\%-21.8\% of the phenotypic variation across environments (Tables 6 and S4; Figure 1). A QTL on Chr. 5A between IWB31050 and IWB54778 at 108.49-112.13 cm was found in four environments (15ABE1, 15ABE2, 15ABE3 and Average environment), explaining $12.3 \%-19.6 \%$ of the phenotypic variation. Additionally, a QTL on Chr. 7A-2 between IWB50481and IWB10274 at 20.02- $21.84 \mathrm{~cm}$ was found in three environments (15ABE1, 15ABE2 and Average environment), explaining $9.9 \%-18.3 \%$ of the phenotypic variation.

Table 6. QTLs for plant height (PH), thousand kernels weight (TKW) and grain yield (GY) detected in the DH population of UI Platinum/SY Capstone.

\begin{tabular}{|c|c|c|c|c|c|c|c|c|c|}
\hline Trial & & QTL $^{a}$ & Peak Marker & LG & Position $b$ & Marker Interval & LOD $^{c}$ & Addd & $\mathrm{R}^{2 \mathrm{e}}$ \\
\hline \multirow[t]{20}{*}{$\mathrm{PH}$} & \multirow[t]{6}{*}{ 15ABE1 } & Q.PH.ui-6A-2.1 & IWB70003 & $6 A-2$ & 110.31 & IWB31050-IWB54778 & 5.2 & 3.36 & 19.6 \\
\hline & & Q.PH.ui-7A-2 & IWB10274 & $7 \mathrm{~A}-2$ & 21.84 & IWB50481-IWB10274 & 2.7 & -2.37 & 10.9 \\
\hline & & Q.PH.ui-2B-1 & IWB42693 & $2 \mathrm{~B}-1$ & 10.01 & IWB10588-IWB42693 & 4.2 & 3.24 & 16.1 \\
\hline & & Q.PH.ui-5B-2.1 & IWB626 & $5 \mathrm{~B}-2$ & 42.9 & IWB36579-IWB18101 & 4.8 & -3.55 & 18.3 \\
\hline & & Q.PH.ui-5B-2.2 & IWB2079 & $5 \mathrm{~B}-2$ & 94.84 & IWB19792-IWB1762 & 3.0 & 2.57 & 11.8 \\
\hline & & Q.PH.ui-5D-3 & IWB17912 & $5 \mathrm{D}-3$ & 0 & IWB17912-IWB63558 & 2.6 & 2.50 & 10.3 \\
\hline & \multirow[t]{5}{*}{ 15ABE2 } & Q.PH.ui-1A & IWB13768 & $1 \mathrm{~A}$ & 96.17 & IWB34600-IWB67661 & 3.7 & 3.12 & 14.3 \\
\hline & & Q.PH.ui-6A-2.1 & IWB70003 & $6 A-2$ & 110.31 & IWB31050-IWB54778 & 3.4 & 3.06 & 13.3 \\
\hline & & Q.PH.ui-6A-2.2 & IWB40830 & $6 \mathrm{~A}-2$ & 118.5 & IWB40830-IWB52007 & 4.0 & 3.32 & 15.6 \\
\hline & & Q.PH.ui-7A-2 & IWB10274 & $7 \mathrm{~A}-2$ & 23.84 & IWB50481-IWB10274 & 2.5 & -2.60 & 9.9 \\
\hline & & Q.PH.ui-2D-1 & IIWB15442 & 2D-1 & 133.01 & IWB15442-IWB60397 & 3.7 & 3.15 & 14.4 \\
\hline & \multirow[t]{3}{*}{ 15ABE3 } & Q.PH.ui-1A & IWB13768 & $1 \mathrm{~A}$ & 96.17 & IWB34600-IWB67661 & 2.7 & 2.41 & 10.7 \\
\hline & & Q.PH.ui- $6 A-2.1$ & IWB70003 & $6 A-2$ & 110.31 & IWB31050-IWB54778 & 3.3 & 2.76 & 12.8 \\
\hline & & Q.PH.ui-1B-1 & Barc80 & 1B-1 & 14 & Barc80-IWB26010 & 4.8 & 3.75 & 18.1 \\
\hline & \multirow[t]{2}{*}{ 16ABE4 } & Q.PH.ui-5B-2.2 & IWB2079 & $5 \mathrm{~B}-2$ & 94.84 & IWB19792-IWB1762 & 3.2 & 2.68 & 12.7 \\
\hline & & Q.PH.ui-7B & IWB72296 & $7 \mathrm{~B}$ & 0 & IWB72296-IWB13270 & 4.3 & 2.99 & 16.6 \\
\hline & \multirow[t]{4}{*}{ Average $^{f}$} & Q.P.Н.иi-6A-2.1 & IWB70003 & $6 A-2$ & 110.31 & IWB31050-IWB54778 & 3.6 & 1.88 & 14.2 \\
\hline & & Q.PH.ui-6A-2.2 & IWB40830 & $6 \mathrm{~A}-2$ & 118.5 & IWB40830-IWB52007 & 3.5 & 1.88 & 13.7 \\
\hline & & Q.PH.ui-7A-2 & IWB10274 & $7 \mathrm{~A}-2$ & 21.84 & IWB50481-IWB10274 & 2.7 & -1.52 & 10.8 \\
\hline & & Q.PH.ui-5B-2.1 & IWB626 & $5 \mathrm{~B}-2$ & 42.9 & IWB36579-IWB18101 & 2.8 & -1.61 & 11.2 \\
\hline \multirow[t]{22}{*}{ TKW } & \multirow[t]{4}{*}{ 15ABE1 } & Q.TKW.ui-2A-1.1 & IWB22006 & $2 \mathrm{~A}-1$ & 37.57 & IWB11289-IWB1047 & 4.1 & -2.45 & 15.7 \\
\hline & & Q.TKW.ui-2A-1.2 & IWB51843 & $2 \mathrm{~A}-1$ & 44.85 & IWB14710-IWB51843 & 2.9 & -2.11 & 11.3 \\
\hline & & Q.TKW.ui-3B-1 & IWB5790 & 3B-1 & 50.52 & IWB5790-IWB10800 & 5.3 & -2.83 & 20.0 \\
\hline & & Q.TKW.ui-5D-1 & IWB71674 & $5 \mathrm{D}-1$ & 34.35 & IWB22695-IWB35177 & 3.4 & 2.16 & 13.2 \\
\hline & \multirow[t]{3}{*}{ 15ABE2 } & Q.TKW.ui-2D-1 & $c f d 73$ & $2 D-1$ & 108.19 & $c f d 73-I W B 1093$ & 3.7 & -2.07 & 14.3 \\
\hline & & Q.TKW.ui-3D-1.1 & IWB35432 & $3 \mathrm{D}-1$ & 43.71 & IWB35432-IWB63148 & 2.6 & -1.70 & 10.2 \\
\hline & & Q.TKW.ui-3D-1.2 & IWB45650 & $3 \mathrm{D}-1$ & 12 & IWB45650-Wms3 & 5.3 & -2.13 & 19.5 \\
\hline & \multirow[t]{5}{*}{ 15ABE3 } & Q.TKW.ui-2B-4 & IWB11240 & $2 \mathrm{~B}-4$ & 101.17 & IWB11240-IWB42141 & 5.4 & -2.54 & 20.4 \\
\hline & & Q.TKW.ui-2D-1 & $c f d 73$ & $2 D-1$ & 108.19 & cfd73-IWB1093 & 3.8 & -1.95 & 14.8 \\
\hline & & Q.TKW.ui-3D-1.1 & IWB35432 & 3D-1 & 43.71 & IWB35432-IWB63148 & 3.7 & -1.88 & 14.2 \\
\hline & & Q.TKW.ui-3D-1.2 & IWB45650 & 3D-1 & 12 & IWB45650-Wms3 & 4.8 & -2.40 & 18.2 \\
\hline & & Q.TKW.ui-5D-1 & IWB71674 & $5 \mathrm{D}-1$ & 34.35 & IWB22695-IWB35177 & 3.3 & 1.74 & 12.8 \\
\hline & \multirow[t]{4}{*}{ 16ABE4 } & Q.TKW.ui-4A-2 & IWB21392 & $4 \mathrm{~A}-2$ & 12 & IWB21392-IWB34577 & 3.9 & 1.79 & 15.2 \\
\hline & & Q.TKW.ui-5A & IWB17401 & $5 \mathrm{~A}$ & 203.25 & IWB17401-IWB56891 & 5.4 & 2.08 & 20.2 \\
\hline & & Q.TKW.ui-2D-1 & $c f d 73$ & $2 D-1$ & 108.19 & $c f d 73-I W B 1093$ & 4.6 & -1.68 & 17.5 \\
\hline & & Q.TKW.ui-2D-1.2 & IWB22901 & 2D-1 & 161.07 & IWB22901-Gwm484 & 5.1 & -2.10 & 19.1 \\
\hline & \multirow[t]{6}{*}{ Average $^{\mathrm{f}}$} & Q.TKW.ui-2A-1.1 & IWB22006 & $2 \mathrm{~A}-1$ & 37.57 & IWB11289-IWB1047 & 7.4 & -1.81 & 26.5 \\
\hline & & Q.TKW.ui-2A-1.2 & IWB51843 & $2 \mathrm{~A}-1$ & 44.85 & IWB14710-IWB51843 & 5.7 & -1.72 & 21.1 \\
\hline & & Q.TKW.ui-2A-1.3 & IWB10540 & $2 \mathrm{~A}-1$ & 108.22 & IWB12554-IWB5752 & 5.4 & 1.48 & 20.1 \\
\hline & & Q.TKW.ui-3B-1 & IWB5790 & 3B-1 & 50.52 & IWB5790-IWB10800 & 2.8 & -1.04 & 10.9 \\
\hline & & Q.TKW.ui-2D-1 & $c f d 73$ & $2 D-1$ & 108.19 & $c f d 73-I W B 1093$ & 5.7 & -1.52 & 21.1 \\
\hline & & Q.TKW.ui-5D-1 & IWB71674 & $5 \mathrm{D}-1$ & 34.35 & IWB22695-IWB35177 & 4.2 & 1.28 & 16.0 \\
\hline \multirow[t]{7}{*}{ GY } & \multirow[t]{4}{*}{ 15ABE1 } & Q.GY.ui-7A-1 & IWB10036 & $7 \mathrm{~A}-1$ & 32.53 & IWB10481-IWB12655 & 4.8 & 0.48 & 18.2 \\
\hline & & Q.GY.ui-5B-2 & IWB2079 & $5 \mathrm{~B}-2$ & 94.84 & IWB19792-IWB1762 & 3.8 & -0.46 & 14.6 \\
\hline & & Q.GY.ui-6B & IWB42306 & $6 B$ & 34.61 & IWB54721-IWB42306 & 4.4 & -0.57 & 16.7 \\
\hline & & Q.GY.ui-3D-1 & IWB35432 & 3D-1 & 43.71 & IWB35432-IWB63148 & 3.2 & -0.39 & 12.7 \\
\hline & \multirow[t]{3}{*}{ 15ABE2 } & Q.GY.ui-2A-1.1 & IWB14710 & $2 \mathrm{~A}-1$ & 41.21 & IWB14710-IWB51843 & 2.5 & -0.37 & 9.9 \\
\hline & & Q.GY.ui-5A & IWB11626 & $5 \mathrm{~A}$ & 120.51 & IWB11626-IWB35454 & 2.9 & -0.40 & 11.3 \\
\hline & & Q.GY.ui-2B-4 & IWB11240 & $2 \mathrm{~B}-4$ & 111.17 & IWB11240-IWB42141 & 3.7 & -2.04 & 14.5 \\
\hline
\end{tabular}


Table 7. QTLs for plant height (PH), thousand kernels weight (TKW) and grain yield (GY) detected in the DH population of UI Platinum/SY Capstone.

\begin{tabular}{|c|c|c|c|c|c|c|c|c|}
\hline Trial & QTL $^{a}$ & Peak Marker & LG & Position $^{b}$ & Marker Interval & LOD $^{c}$ & Addd & $\mathrm{R}^{2 \mathrm{e}}$ \\
\hline \multirow{3}{*}{ 15ABE3 } & Q.GY.ui-6B & IWB42306 & $6 B$ & 34.61 & IWB54721-IWB42306 & 5.1 & -0.56 & 19.2 \\
\hline & Q.GY.ui-2A-1.1 & IWB14710 & $2 \mathrm{~A}-1$ & 41.21 & IWB14710-IWB51843 & 2.7 & -0.32 & 10.8 \\
\hline & Q.GY.ui-3B-1 & IWB58000 & 3B-1 & 169.8 & IWB58000-IWB67913 & 4.0 & -0.87 & 15.4 \\
\hline \multirow{6}{*}{ 16ABE4 } & Q.GY.ui-5D-3 & Barc320 & $5 \mathrm{D}-3$ & 30.31 & Barc320-IWB58388 & 4.8 & 0.45 & 18.4 \\
\hline & Q.GY.ui-1A.1 & IWB13768 & $1 \mathrm{~A}$ & 96.17 & IWB34600-IWB67661 & 2.9 & -0.78 & 11.6 \\
\hline & Q.GY.ui-1A.2 & IWB52960 & $1 \mathrm{~A}$ & 110.81 & IWB78643-IWB31771 & 5.2 & 1.16 & 19.6 \\
\hline & Q.GY.ui-6A-2 & IWB34744 & $6 \mathrm{~A}-2$ & 155.47 & IWB34744-IWB199 & 2.7 & -0.39 & 10.6 \\
\hline & Q.GY.ui-6B & IWB42306 & $6 B$ & 34.61 & IWB54721-IWB42306 & 4.3 & -0.60 & 16.6 \\
\hline & Q.GY.ui-1D-1 & IWB42629 & 1D-1 & 11.94 & IWB42629-IWB80211 & 5.5 & 0.59 & 20.4 \\
\hline \multirow[t]{8}{*}{ Average $^{f}$} & Q.GY.ui-2A-1.1 & IWB14710 & $2 \mathrm{~A}-1$ & 41.21 & IWB14710-IWB51843 & 3.6 & 0.20 & 13.8 \\
\hline & Q.GY.ui-2A-1.2 & IWB22006 & $2 \mathrm{~A}-1$ & 37.57 & IWB11289-IWB1047 & 3.1 & -0.19 & 12.2 \\
\hline & Q.GY.ui-5A & IWB11626 & $5 \mathrm{~A}$ & 120.51 & IWB11626-IWB35454 & 2.6 & -0.18 & 10.4 \\
\hline & Q.GY.ui-1B-1 & Barc80 & $1 \mathrm{~B}-1$ & 4 & Barc80-IWB26010 & 5.6 & 0.32 & 20.8 \\
\hline & Q.GY.ui-1B-2 & Barc152 & $1 \mathrm{~B}-2$ & 59.02 & Barc152-IWB30286 & 2.7 & 0.18 & 10.6 \\
\hline & Q.GY.ui-2B-2.1 & IWB15495 & $2 \mathrm{~B}-2$ & 30.98 & IWB14855-IWB50665 & 4.2 & -0.23 & 16.3 \\
\hline & Q.GY.ui-2B-2.2 & IWB10507 & $2 \mathrm{~B}-2$ & 36.44 & IWB10507-IWB17472 & 4.4 & -0.23 & 16.8 \\
\hline & Q.GY.ui-6B & IWB42306 & $6 B$ & 34.61 & IWB54721-IWB42306 & 2.8 & -0.18 & 11.2 \\
\hline
\end{tabular}

15ABE1, 15ABE2, 15ABE3: trial 1, 2 and 3 in Aberdeen in 2015, respectively; 16ABE4, trial 4 in Aberdeen in 2016. a ui, university of Idaho. Different QTL on the same linkage group was indicated with 1 and 2 after the period following the linkage group. ${ }^{b}$ Position of QTL located on linkage group, as CM distance from the top of each linkage group. ${ }^{\mathrm{c}}$ A LOD threshold of 2.5 was used for declaration of QTL. d Positive "additive effect" indicated effect from SY Capstone, negative "additive effect" indicated effect from UI Platinum. " Percentage of phenotypic variation explained by the QTL. ${ }^{\mathrm{f}}$ Average data across the four or five environments was used. LG, linkage group. Italic and bold showed the stable QTL for traits.

For TKW, 15 QTL were detected on the homologous chromosome groups 2 and 5 as well as on Chr. 4A-2, 3B-1, 3D-1, and 7B, explaining $10.2 \%-31.3 \%$ of the phenotypic variation across environments (Tables 6 and S4; Figure 1). A QTL on Chr. 2D-1 between $c f d 73$ and IWB1093 at 108.19-110.01 cm was found four environments (15ABE3, 15ABE3, 16ABE4 and Average environment), explaining $14.3 \%-21.1 \%$ of the phenotypic variation. Additionally, a QTL on Chr. 5D-1 between IWB 22695 and IWB35177 at 33.44-36.17 cm was found in three environments (15ABE1, 15ABE3 and Average environment), respectively, explaining $10.2 \%-16.2 \%$ and $18.2 \%-31.1 \%$ of the phenotypic variation, respectively.

For GY, 18 QTL were detected on the homologous chromosome groups 1 and 5 as well as on Chr. 2A-1, 6A-2, 7A-1, 2B-2, 2B-4, 3B-1, 6B, and 3D-1, explaining 9.8\%-20.8\% of the phenotypic across environments (Tables 6 and S4; Figure 1). A stable QTL on Chr. 6B between IWB54721 and IWB42306 at 26.2-32.61 cm was found in four environments (15ABE1, 15ABE2, 16ABE4 and Average environment), explaining $11.2 \%-19.2 \%$ of the phenotypic variation. In addition, one QTL on Chr. 2A-1 between IWB14710 and IWB51843 at 41.21-44.85 cm was found in three environments (15ABE2, 15ABE3 and Average environment), each explaining $9.9 \%-13.8 \%$ of the phenotypic variation.

\section{Discussion}

Improving GY is an essential objective in wheat breeding programs worldwide. Physiological traits, as well as PH and TKW, are critical elements of GY. The wide use of molecular markers has made possible the study of quantitative traits $[9,11,23]$ and detect QTL that allow the development of high-yield cultivars via MAS. Previous studies identified numerous QTL associated with yield-related traits in various populations $[1,9,10,19-21,27]$. However, the genetic architecture of these traits needs further research. In this study, a DH population was phenotyped for CT (CTa, CTg1, and CTg2), CCI (CCIa, CCIg1, and CCIg2), PH, TKW, and GY under irrigated conditions and genotyped with SNP markers and SSRs to better understand the genetic control of the studied traits.

\subsection{Phenotypic Evaluation}

In the present study, $\mathrm{PH}$ and TKW showed high heritability; CCIa, CCIg1, and CCIg2 moderate heritability; whereas $\mathrm{CTa}, \mathrm{CTg} 1$, and $\mathrm{CTg} 2$ low heritability, results that were in agreement with those reported in previous studies $[10,11,47]$. Environmental diversity affects CT heritability, which ranges from 
low under heat or water-stressed conditions [9] to high under irrigated conditions [28]. GY is a complex trait highly affected by the environment and thus, has low heritability [48]. However, in the present study, the heritability of GY was higher than that reported previously [49]. The higher heritability could be attributed to the lower error variance. Overall, our results suggested that PH and TKW could be used for early generation breeding because of their high heritability, whereas $\mathrm{CT}$ and $\mathrm{CCI}$ could be also used due to their strong correlation with GY, similarly as reported by Zhang (2013) [49].

\subsection{Linkage Map Construction}

Molecular markers have been widely applied for genetic map construction, QTL detection, gene cloning, and MAS [15]. SNP markers are the most abundant polymorphisms in plant genomes [32], and thus, allow the high-throughput genotyping compared with previous marker platforms [30,31]. In the present study, we used the $90 \mathrm{~K}$ iSelect SNP array combined with SSRs to genotype a spring wheat DH population derived from a cross between UI and SY. Using 9687 polymorphic SNPs, 9330 previously mapped [31] and 357 newly mapped, we constructed a genetic map of 43 LGs that represented all the 21 chromosomes of bread wheat (Table S2) and had a total length of $3633.19 \mathrm{~cm}$ and an average marker density of $0.37 \mathrm{~cm}$ (Table S2). Our linkage map had a better marker distribution and a higher density compared with those reported in previous studies [11,23,50-55]. However, some gaps were observed in Chr. 1A and 1B-1. The A and B genomes were mapped by 4320 and 4367 markers, respectively, whereas the D genome by 1046 markers (Table S2), suggesting that the former genomes included more polymorphisms. These results were in agreement with those reported in previous studies $[11,31,56]$.

The average number of markers mapped per chromosome was 463.5, ranging from 15 on Chr. 3D to 1125 on Chr. 6A (Table S2). Although $89.4 \%$ of mapped SNPs displayed redundancy, only $10.6 \%$ was used to construct the linkage map, similarly as in previous studies, since many SNPs were co-located at the same loci [11,57].

\subsection{QTL Mapping}

Previous studies reported a large number of QTL for several physiological and agronomic traits under different conditions [9-11,27,28]. In the present study, we detected QTL for CTa, CTg1, and CTg2 on Chr. 2B, 4B, 5B-2, and 6B (Tables 4 and S3). Of these, the stable QTL Q.CTa.ui-4B.1 on Chr. 4B at 27.83-30.56 cm (Table 4; Figure 1) was different from that reported by Gao et al. (2016) [10] linked to RAC875_c6749_954 at $42.0 \mathrm{~cm}$; the stable QTL Q.CTg1.ui-5B-2.1 on Chr. 5B at 10.96-13.69 $\mathrm{cm}$ was different from that reported by [21] at $52.4-55.7 \mathrm{~cm}$ and from that reported by Gao et al. (2016) [10] close to wsnp_Ex_c10842_17637744 at $54.1 \mathrm{~cm}$; and the stable QTL Q.CTg2.ui-6B.1 on Chr. $6 \mathrm{~B}$ at $87.2-88.11 \mathrm{~cm}$ has not been reported in any previous studies.

We also detected QTL for CCIa, CCIg1, and CCIg2 on Chr. 2D-1, 3A-2, 4A-1, and 5A (Tables 5 and S3). Of these, the QTL Q.CCIg1.ui-5A.1 on Chr. 5A tightly linked to IWB14149 at $91.35 \mathrm{~cm}$ was different from that reported by Gao et al. (2015) [11] at $72 \mathrm{~cm}$; and the minor QTL Q.CCIg2.ui-5A.1 of Chr. 5A has not been reported in any previous studies.

$\mathrm{PH}$ is considered as a complex trait, consisted of internode and spike length [9,58]. Rht-B1b and Rht-D1b (previously known as Rht1 and Rht2) on Chr. 4B and 4D, respectively [43], that result in semi-dwarfism are widely used in wheat breeding. In the present study, both UI and SY carried $R h t-D 1 b$, but not Rht-B1b, and thus, no QTL for PH were identified on Chr. 4B and 4D. However, we detected the stable QTL Q.PH.ui-6A-2.1 on Chr. 6A-2 that has not been reported in any previous studies as well as two QTL on Chr. 7A-2 and 5B-2 that were consistently detected in two environments, explaining $9.9 \%-18.3 \%$ of the phenotypic variation (Table 7). Additionally, the minor QTL Q.PH.ui-5A on Chr. 5A at $41.26 \mathrm{~cm}$ was likely the same as that previously reported by Lopes et al. (2013) [9] and Gao et al. (2015) [11]. Additionally, two minor QTL identified for PH and GY on Chr. 1A and 1B-1 might indicate that other genes for PH probably have minor, but significant effects on GY. 
Previous studies reported major QTL on Chr. 5A, affecting adaptability and productivity [59-61], as well as QTL for yield-related traits on the homologous chromosome groups of 5, 6, and 7 [62]. In the present study, the stable QTL Q.TKW.ui-2D-1 on Chr. 2D-1 between cfd73 and IWB1093 at 108.19-110.01 cm (Table 5; Figure 1) and the QTL Q.TKW.ui-3D-1.2 on Chr. 3D-1 at 0-22.27 cm were different from those previously reported for TKW by Gao et al. (2015) [11].

QTL for yield and yield-related traits have been identified on Chr. 4A under drought and other stress conditions $[9,28,63,64]$. In the present study, we detected QTL for GY on Chr. 1A, 1B-1, 1B-2, 2A-1, 2B-2, 2B-4, 3B-1, 3D, 5A, 5B-2, 5D-3, 6A-2, 6B, and 7A-1 across all environments (Table S3), but not any on Chr. 4A. Differences in QTL locations could be attributed to the environmental conditions, which were more favorable for wheat production in the present study. However, we detected QTL on Chr. 4A-1 with pleiotropic effects on CCIa, CCIg1, and CCIg2. The stable QTL Q.GY.ui-6B on Chr. 6B at 26.21-32.61 cm (Table 7; Figure 1) was different from that reported by Zhang (2013) [49] at 51.9-57.3 cm.

Previous studies reported QTL clusters for various traits $[10,11,65]$. Here, we detected 26 QTL clusters for more than two traits (Table 8; Figure 1) on Chr. 1A, 2A-1(2), 1B-1, 2B-2, 2B-4, 5B-2, 1D-2, and 3D-1. Pleiotropic QTL for GY and PH were detected on Chr. 1A and 1B-1, and analysis of their allelic effects showed that a decrease in GY due to the presence of the SY allele was accompanied by an increase in $\mathrm{PH}$ (data not shown). Additionally, pleiotropic QTL for GY and KWT were found on Chr. 2A-1 and 2B-4 (2), and analysis of their allelic effects showed that the presence of the UI allele on Chr. 2A-1 increased TKW and GY (data not shown). Therefore, pleiotropic QTL confirmed the positive correlation between TKW and GY (Table 3). Pleiotropic QTL for GY and CTa and also for CTa and CTg2 were detected on Chr. 2B-2 and 4B (2), respectively. Analysis of their allelic effects showed that the presence of the UI allele reduced CTa and CTg2, but increased GY (data not shown). Thus, pleiotropic QTL on Chr. 2B confirmed the negative correlation between GY and CTa, whereas those on Chr. 4B the positive correlation between CTa and CTg2 (Table 3).

Table 8. Summary of pleiotropic QTLs identified in the SY Capstone/UI Platinum DH population.

\begin{tabular}{|c|c|c|c|c|}
\hline Linkage Group & Cluster & Traits & Marker Interval & Position $^{a}$ \\
\hline $1 \mathrm{~A}$ & 1 & PH, GY & IWB34600-IWB67661 & $94.35-97.08$ \\
\hline \multirow[t]{2}{*}{$1 \mathrm{~B}-1$} & 1 & PH, GY & Barc80-IWB26010 & $0-18.01$ \\
\hline & 2 & CTg1, CTg2, CCIg2 & IWB10780-IWB58775 & $127.09-128$ \\
\hline 1D-1 & 1 & CCIg1, GY & IWB42629-IWB80211 & $11.94-23.0$ \\
\hline \multirow[t]{2}{*}{$2 \mathrm{~A}-1$} & 1 & TKW, GY & IWB11289-IWB1047 & $36.66-38.48$ \\
\hline & 2 & TKW, GY & IWB56873-IWB51843 & $43.03-44.85$ \\
\hline \multirow[t]{2}{*}{$2 \mathrm{~B}-2$} & 1 & CTg2, CCIg1 & IWB22675-IWB11319 & 20.97-25.52 \\
\hline & 2 & CTa, GY & IWB11092-IWB17472 & $41.9-43.72$ \\
\hline $2 B-3$ & 1 & CTg1, CCIa & IWB18944-Wmc149 & $0-24.52$ \\
\hline $2 \mathrm{~B}-4$ & 1 & TKW, GY & IWB11240-IWB42141 & $91.17-111.28$ \\
\hline \multirow[t]{2}{*}{$2 \mathrm{D}-1$} & 1 & CTg2, TKW & cfd73-IWB1093 & $108.19-110.01$ \\
\hline & 2 & $\begin{array}{c}\text { CCIa, CCIg1, } \\
\text { CCIg2, PH }\end{array}$ & IWB15442-IWB60397 & 133.01-134.83 \\
\hline $3 \mathrm{~A}-1$ & 1 & $\mathrm{CTg} 2, \mathrm{CCla}$ & IWB33546-IWB14315 & $0-5.58$ \\
\hline $3 \mathrm{~B}-1$ & 1 & CCIg2, TKW & IWB5790-IWB10800 & $50.52-54.16$ \\
\hline \multirow[t]{2}{*}{$3 \mathrm{D}-1$} & 1 & TKW, GY & IWB35432-IWB63148 & $43.71-45.53$ \\
\hline & 2 & CCIa, TKW & IWB45650-Wms3 & $0-22.27$ \\
\hline $4 \mathrm{~A}-1$ & 1 & $\begin{array}{c}\text { CCIa, CCIg1, } \\
\text { CCIg2 }\end{array}$ & IWB37346-IWB10035 & $78.04-79.86$ \\
\hline \multirow[t]{2}{*}{$4 \mathrm{~B}$} & 1 & $\mathrm{CTa}, \mathrm{CTg} 2$ & IWB20226-IWB57507 & $27.83-30.56$ \\
\hline & 2 & CTa, CTg2 & IWB12144-IWB35486 & $33.29-34.2$ \\
\hline \multirow[t]{2}{*}{$5 \mathrm{~A}$} & 1 & CCIg1, CCIg2 & IWB14149-IWB10765 & $91.35-93.17$ \\
\hline & 2 & CCIg1, CCIg2 & IWB21197-IWB17983 & $165.38-169.93$ \\
\hline \multirow[t]{4}{*}{$5 B-2$} & 1 & CTg1, CTg2 & IWB80334-IWB29709 & $10.96-13.69$ \\
\hline & 2 & CTa, CCIg2 & IWB36033-IWB19921 & $18.24-20.97$ \\
\hline & 3 & $\mathrm{CTa}, \mathrm{PH}$ & IWB36579-IWB18101 & $41.08-44.72$ \\
\hline & 4 & PH, GY & IWB19792-IWB1762 & 92.11-95.75 \\
\hline $6 \mathrm{~B}$ & 1 & $\mathrm{CTa}, \mathrm{CTg} 1$ & IWB75959-IWB10604 & $0-3.64$ \\
\hline
\end{tabular}

a Position of QTL located on chromosome, as CM distance from the top of each linkage group. PH, plant height; GY, grain yield; TKW, thousand kernels weight; $\mathrm{CTa}, \mathrm{CTg} 1, \mathrm{CTg} 2$, canopy temperature during anthesis, mid- and late grain-filling stage, respectively; CCIa, CCIg1, CCIg2, chlorophyll content index during anthesis, mid- and late grain-filling stage respectively. 


\subsection{Practical Applications in Wheat Breeding}

Wheat GY is a complex, quantitative trait, highly affected by the environment; thus, early generation selection for high yield is usually not effective. In the present study, we suggested that CT, CCIa, PH, and TKW could be used for increasing GY using stable QTL, similarly as reported in previous studies $[9,11]$. GY was significantly positively correlated CCIa, $\mathrm{PH}$, and TKW, negatively correlated with $\mathrm{CTa}, \mathrm{CTg} 1$, and $\mathrm{CTg} 2$; therefore, these traits could be used for early generation and early growth stage selections. Additionally, some stable QTL, such as Q.CTa.ui-4B.1, Q.CTg1.ui-5B-2.1, Q.CTg2.ui-6B.1, Q.PH.ui-6A-2.1, Q.TKW.ui-2D-1, and Q.GY.ui-6B, could be applied in MAS for yield improvement after validation.

\section{Conclusions}

Genome-wide linkage mapping was used to identify QTLs associated with CTa, CTg1, CTg2, CCIa, CCIa, CCIg1, CCIg2, PH, TKW, and GY using high-density genetic linkage map in a spring wheat DH population derived from a cross between 'UI Platinum' and 'SY Capstone'. The DH population was genotyped using the wheat $90 \mathrm{~K}$ iSelect platform and SSRs. The linkage map was constructed using a total of 9687 SNP marker and 44 SSRs, with whole linkage map of $3594.0 \mathrm{~cm}$ and marker density of $0.37 \mathrm{~cm}$ between adjacent markers. A total of 116 QTLs were detected for the nine traits on 33 linkage groups, representing the whole 21 wheat chromosomes, except for Chr. 7D. Among these QTLs, 71 QTL were for CT and CCI, and 45 QTL were for PH, TKW, and GY. Six QTLs were consistently detected more than three irrigated environments, called as stable QTL. 26 QTL clusters were identified for more than two traits, and four QTL-rich chromosome regions on these traits were found on chromosomes 4A-1, 1B-1, 5B-2, and 2D-1. These QTLs, QTL clusters and linked molecular markers may be very useful in assisted selection for improving grain yield in spring wheat breeding.

Supplementary Materials: The following are available online at http:/ /www.mdpi.com/2073-4395/8/5/60/s1, Figure S1: The frequency distribution of canopy temperature (CT) (A), chlorophyll content index (CCI) (B), plant height (PH), thousand kernels weight (TKW) and grain yield (GY) (C) in the DH population from UI Platinum/SY Capstone in the four environments, Table S1: Phenotypic variations for physiological traits, plant height $(\mathrm{PH})$, thousand kernel weight (TKW) and grain yield (GY) in UI Platinum and SY Capstone and their DH population across four trial, Table S2: Summary of the genetic linkage maps constructed with SNP, SSR and STS markers using DH population derived from UI Platinum / SY Capstone, Table S3: The names and positions of 357 newly mapped SNP markers, Table S4: Summary of QTLs detected for physiological traits, plant height (PH), thousand kernels weight (TKW) and grain yield (GY) in the UI Platinum/SY Capstone DH population.

Author Contributions: Y.L. analyzed the data and developed the manuscript. R.W. and Y.-g.H. contributed to the manuscript. J.C. was the supervisor of Y.L., who designed and oversaw overall activities of the experiments and manuscript development.

Acknowledgments: This project was supported by the National Research Initiative Competitive Grants 2011-68002-30029 and 2017-67007-25939 from the USDA National Institute of Food and Agriculture; the Idaho Wheat Commission; the Idaho Agricultural Experimental Station Hatch Projects; and the China Scholarship Council under Grant 201406300139. The authors would like to thank Justin Wheeler, Natalie Klassen, Weidong Zhao, and Dr. Jun Wu for their technical assistance.

Conflicts of Interest: The authors declare no conflict of interest.

\section{References}

1. Edae, E.A.; Byrne, P.F.; Haley, S.D.; Lopes, M.S.; Reynolds, M.P. Genome-wide association mapping of yield and yield components of spring wheat under contrasting moisture regimes. Theor. Appl. Genet. 2014, 127, 791-807. [CrossRef] [PubMed]

2. Reynolds, M.; Foulkes, J.; Furbank, R.; Griffiths, S.; King, J.; Murchie, E.; Parry, M.; Slafer, G. Achieving yield gains in wheat. Plant Cell Environ. 2012, 35, 1799-1823. [CrossRef] [PubMed]

3. Fedoroff, N.V.; Battisti, D.S.; Beachy, R.N.; Cooper, P.J.M.; Fischhoff, D.A.; Hodges, C.N.; Knauf, V.C.; Lobell, D.; Mazur, B.J.; Molden, D. Radically rethinking agriculture for the 21st century. Science 2010, 327, 833-834. [CrossRef] [PubMed] 
4. Stamp, P.; Visser, R. The twenty-first century, the century of plant breeding. Euphytica 2012, 186, 585-591. [CrossRef]

5. Yang, Z.; Bai, Z.; Li, X.; Wang, P.; Wu, Q.; Yang, L.; Li, L.; Li, X. Snp identification and allelic-specific pcr markers development for tagw2, a gene linked to wheat kernel weight. Theor. Appl. Genet. 2012, 125, 1057-1068. [CrossRef] [PubMed]

6. Arbuckle, J.G.; Prokopy, L.S.; Haigh, T.; Hobbs, J.; Knoot, T.; Knutson, C.; Loy, A.; Mase, A.S.; McGuire, J.; Morton, L.W.; et al. Climate change beliefs, concerns, and attitudes toward adaptation and mitigation among farmers in the midwestern united states. Clim. Chang. 2013, 117, 943-950. [CrossRef]

7. Li, H.W.; Tong, Y.P.; Li, B.; Jing, R.L.; Lu, C.M.; Li, Z.S. Genetic analysis of tolerance to photo-oxidative stress induced by high light in winter wheat (Triticum aestivum L.). J. Genet. Genom. 2010, 37, 399-412. [CrossRef]

8. Li, P.; Chen, J.L.; Wu, P.T. Evaluation of grain yield and three physiological traits in 30 spring wheat genotypes across three irrigation regimes. Crop Sci. 2012, 52, 110-121. [CrossRef]

9. Lopes, M.S.; Reynolds, M.P.; McIntyre, C.L.; Mathews, K.L.; Kamali, M.R.J.; Mossad, M.; Feltaous, Y.; Tahir, I.S.A.; Chatrath, R.; Ogbonnaya, F.; et al. QTL for yield and associated traits in the seri/babax population grown across several environments in Mexico, in the West Asia, North Africa, and South Asia regions. Theor. Appl. Genet. 2013, 126, 971-984. [CrossRef] [PubMed]

10. Gao, F.M.; Liu, J.D.; Yang, L.; Wu, X.X.; Xiao, Y.G.; Xia, X.C.; He, Z.H. Genome-wide linkage mapping of QTL for physiological traits in a chinese wheat population using the 90k snp array. Euphytica 2016, 209, 789-804. [CrossRef]

11. Gao, F.M.; Wen, W.E.; Liu, J.D.; Rasheed, A.; Yin, G.H.; Xia, X.C.; Wu, X.X.; He, Z.H. Genome-wide linkage mapping of QTL for yield components, plant height and yield-related physiological traits in the Chinese wheat cross Zhou 8425b/Chinese Spring. Front. Plant Sci. 2015, 6, 1099. [CrossRef] [PubMed]

12. Reynolds, M.P.; Nagarajan, S.; Razzaque, M.A.; Ageeb, O.A.A. Heat tolerance. In Application of Physiology in Wheat Breeding; Reynolds, M.P., Ortiz-Monasterio, J.I., McNab, A., Eds.; CIMMYT: Texcoco, Mexico, 2001; pp. 124-135.

13. Johnw, S.; Mjohn, F.; James, S.; Michelle, L.; Lesleyj, F.; Wang, Y.; Matteo, C. Dissecting gene x environmental effects on wheat yields via QTL and physiological analysis. Euphytica 2007, 154, 401-408.

14. Zhu, C.; Gore, M.; Buckler, E.S.; Yu, J. Status and prospects of association mapping in plants. Plant Genome 2008, 1, 5-20. [CrossRef]

15. Collard, B.C.; Mackill, D.J. Marker-assisted selection: An approach for precision plant breeding in the twenty-first century. Philos. Trans. R. Soc. Lond. 2008, 363, 557-572. [CrossRef] [PubMed]

16. Holland, J.B. Genetic architecture of complex traits in plants. Curr. Opin. Plant Biol. 2007, 10, $156-161$. [CrossRef] [PubMed]

17. Mcintyre, C.L.; Mathews, K.L.; Rattey, A.; Chapman, S.C.; Drenth, J.; Ghaderi, M.; Reynolds, M.; Shorter, R. Molecular detection of genomic regions associated with grain yield and yield-related components in an elite bread wheat cross evaluated under irrigated and rainfed conditions. Theor. Appl. Genet. 2010, 120, 527-541. [CrossRef] [PubMed]

18. Bogard, M.; Jourdan, M.; Allard, V.; Martre, P.; Perretant, M.R.; Ravel, C.; Heumez, E.; Orford, S.; Snape, J.; Griffiths, S. Anthesis date mainly explained correlations between post-anthesis leaf senescence, grain yield, and grain protein concentration in a winter wheat population segregating for flowering time QTLs. J. Exp. Bot. 2011, 62, 3621. [CrossRef] [PubMed]

19. Bogard, M.; Ravel, C.; Paux, E.; Bordes, J.; Balfourier, F.; Chapman, S.C.; Le, G.J.; Allard, V. Predictions of heading date in bread wheat (Triticum aestivum L.) using QTL-based parameters of an ecophysiological model. J. Exp. Bot. 2014, 65, 5849-5865. [CrossRef] [PubMed]

20. Bennett, D.; Izanloo, A.; Reynolds, M.; Kuchel, H.; Langridge, P.; Schnurbusch, T. Genetic dissection of grain yield and physical grain quality in bread wheat (Triticum aestivum L.) under water-limited environments. Theor. Appl. Genet. 2012, 125, 255-271. [CrossRef] [PubMed]

21. Bennett, D.; Reynolds, M.; Mullan, D.; Izanloo, A.; Kuchel, H.; Langridge, P.; Schnurbusch, T. Detection of two major grain yield QTL in bread wheat (Triticum aestivum L.) under heat, drought and high yield potential environments. Theor. Appl. Genet. 2012, 125, 1473-1485. [CrossRef] [PubMed]

22. Wu, X.; Chang, X.; Jing, R. Genetic insight into yield-associated traits of wheat grown in multiple rain-fed environments. PLoS ONE 2012, 7, e31249. [CrossRef] [PubMed] 
23. Zhang, J.; Chen, J.; Chu, C.; Zhao, W.; Wheeler, J.; Souza, E.J.; Zemetra, R.S. Genetic dissection of QTL associated with grain yield in diverse environments. Agronomy 2014, 4, 556-578. [CrossRef]

24. Sukumaran, S.; Dreisigacker, S.; Lopes, M.; Chavez, P.; Reynolds, M.P. Genome-wide association study for grain yield and related traits in an elite spring wheat population grown in temperate irrigated environments. Theor. Appl. Genet. 2015, 128, 353-363. [CrossRef] [PubMed]

25. Quarrie, S.; Pekic, Q.S.; Radosevic, R.; Rancic, D.; Kaminska, A.; Barnes, J.D.; Leverington, M.; Ceoloni, C.; Dodig, D. Dissecting a wheat QTL for yield present in a range of environments: From the QTL to candidate genes. J. Exp. Bot. 2006, 57, 2627-2637. [CrossRef] [PubMed]

26. Ma, L.; Zhou, E.; Huo, N.; Zhou, R.; Wang, G.; Jia, J. Genetic analysis of salt tolerance in a recombinant inbred population of wheat (Triticum aestivum L.). Euphytica 2007, 153, 109-117. [CrossRef]

27. Rebetzke, G.J.; Rattey, A.R.; Farquhar, G.D.; Richards, R.A.; Condon, A.G. Genomic regions for canopy temperature and their genetic association with stomatal conductance and grain yield in wheat. Funct. Plant Biol. 2013, 40, 14-33. [CrossRef]

28. Pinto, R.S.; Reynolds, M.P.; Mathews, K.L.; Mcintyre, C.L.; Olivaresvillegas, J.J.; Chapman, S.C. Heat and drought adaptive QTL in a wheat population designed to minimize confounding agronomic effects. Theor. Appl. Genet. 2010, 121, 1001-1021. [CrossRef] [PubMed]

29. Vijayalakshmi, K.; Fritz, A.K.; Paulsen, G.M.; Bai, G.; Pandravada, S.; Gill, B.S. Modeling and mapping QTL for senescence-related traits in winter wheat under high temperature. Mol. Breed. 2010, 26, 163-175. [CrossRef]

30. Akhunov, E.; Nicolet, C.; Dvorak, J. Single nucleotide polymorphism genotyping in polyploid wheat with the illumina goldengate assay. Theor. Appl. Genet. 2009, 119, 507-517. [CrossRef] [PubMed]

31. Wang, S.; Wong, D.; Forrest, K.; Allen, A.; Chao, S.; Huang, B.E.; Maccaferri, M.; Salvi, S.; Milner, S.G.; Cattivelli, L. Characterization of polyploid wheat genomic diversity using a high-density 90,000 single nucleotide polymorphism array. Plant Biotechnol. J. 2014, 12, 787-796. [CrossRef] [PubMed]

32. Gupta, P.K.; Rustgi, S.; Mir, R.R. Array-based high-throughput DNA markers for crop improvement. Heredity 2008, 101, 5-18. [CrossRef] [PubMed]

33. Zhai, S.; He, Z.; Wen, W.; Jin, H.; Liu, J.; Zhang, Y.; Liu, Z.; Xia, X. Genome-wide linkage mapping of flour color-related traits and polyphenol oxidase activity in common wheat. Theor. Appl. Genet. 2015, 129, 377-394. [CrossRef] [PubMed]

34. Cabral, A.L.; Jordan, M.C.; Mccartney, C.A.; You, F.M.; Humphreys, D.G.; Maclachlan, R.; Pozniak, C.J. Identification of candidate genes, regions and markers for pre-harvest sprouting resistance in wheat (Triticum aestivum L.). BMC Plant Biol. 2014, 14, 340. [CrossRef] [PubMed]

35. Su, Z.; Jin, S.; Lu, Y.; Zhang, G.; Chao, S.; Bai, G. Single nucleotide polymorphism tightly linked to a major QTL on chromosome 7a for both kernel length and kernel weight in wheat. Mol. Breed. 2016, 36, 1-11. [CrossRef]

36. Chen, J.; Wheeler, J.; O’Brien, K.; Zhao, W.; Klassen, N.; Zhang, J.; Bowman, B.; Wang, Y.; Jackson, C.; Marshall, J.M. Registration of 'ui platinum' hard white spring wheat. J. Plant Regist. 2015, 10, 36-40. [CrossRef]

37. Marshall, J.; Jackson, C.; Shelman, T.; Jones, L.; O’Brien, K. Small Grains Report South Central and South Eastern Idaho Cereal Research and Extension Program. 2011. Available online: https:/ /www.cals.uidaho. edu/edcomm/pdf/RES/RES180.pdf (accessed on 31 January 2012).

38. Laurie, D.A.; Bennett, M.D. Wheat x maize hybridization. Can. J. Genet. Cytol. 1986, 28, 313-316. [CrossRef]

39. Miller, T.D. Growth Stages of Wheat: Identification and Understanding Improve Crop Management; SCS-1999-16; Texas Agricultural Extension Service, the Texas A\&M University System: College Station, TX, USA, 1996.

40. Liu, Y.; Bowman, B.; Hu, Y.G.; Liang, X.; Zhao, W.; Wheeler, J.; Klassen, N.; Bockelman, H.; Bonman, J.; Chen, J. Evaluation of agronomic traits and drought tolerance of winter wheat accessions from the USDA-ARS national small grains collection. Agronomy 2017, 7, 51. [CrossRef]

41. Aldrich, J.; Cullis, C.A. Rapd analysis in flax: Optimization of yield and reproducibility using klen taq 1 DNA polymerase, chelex 100, and gel purification of genomic DNA. Plant Mol. Biol. Rep. 1993, 11, 128-141. [CrossRef]

42. Chen, J.; Chu, C.; Souza, E.J.; Guttieri, M.J.; Chen, X.; Xu, S.; Hole, D.; Zemetra, R. Genome-wide identification of QTL conferring high-temperature adult-plant (HTAP) resistance to stripe rust (Puccinia striiformis $\mathrm{f}$. Sp. Tritici) in wheat. Mol. Breed. 2012, 29, 791-800. [CrossRef] 
43. Ellis, M.H.; Rebetzke, G.J.; Azanza, F.; Richards, R.A.; Spielmeyer, W. Molecular mapping of gibberellinresponsive dwarfing genes in bread wheat. Theor. Appl. Genet. 2005, 111, 423-430. [CrossRef] [PubMed]

44. Ellis, H.; Spielmeyer, W.; Gale, R.; Rebetzke, J.; Richards, A. "Perfect" markers for the rht-b1b and rht-d1b dwarfing genes in wheat. Theor. Appl. Genet. 2002, 105, 1038-1042. [PubMed]

45. Islamovic, E.; Obert, D.E.; Oliver, R.E.; Marshall, J.M.; Miclaus, K.J.; Hang, A.; Chao, S.; Lazo, G.R.; Harrison, S.A.; Ibrahim, A. A new genetic linkage map of barley (hordeum vulgare L.) facilitates genetic dissection of height and spike length and angle. Field Crops Res. 2013, 154, 91-99. [CrossRef]

46. Harrisshultz, K.R.; Davis, R.F.; Knoll, J.E.; Anderson, W.; Wang, H. Inheritance and identification of a major quantitative trait locus (QTL) that confers resistance to meloidogyne incognita and a novel QTL for plant height in sweet sorghum. Phytopathology 2015, 105, 1522-1528. [CrossRef] [PubMed]

47. Reynolds, M.P.; Pierre, C.S.; Saad, A.S.I.; Vargas, M.; Condon, A.G. Evaluating potential genetic gains in wheat associated with stress-adaptive trait expression in elite genetic resources under drought and heat stress. Crop Sci. 2007, 47, S172. [CrossRef]

48. Jackson, P.; Robertson, M.; Cooper, M.; Hammer, G. The role of physiological understanding in plant breeding: From a breeding perspective. Field Crops Res. 1996, 49, 11-37. [CrossRef]

49. Zhang, J. Quantitative Trait Locus Mapping of Agronomic, Physiological, and End-Use Quality Traits of Common Wheat (T. aestivum). Ph.D. Thesis, University of Idaho, Moscow, ID, USA, September 2013.

50. Marone, D.; Laidò, G.; Gadaleta, A.; Colasuonno, P.; Ficco, D.B.M.; Giancaspro, A.; Giove, S.; Panio, G.; Russo, M.A.; Vita, P.D. A high-density consensus map of a and b wheat genomes. Theor. Appl. Genet. 2012, 125, 1619-1638. [CrossRef] [PubMed]

51. Azadi, A.; Mardi, M.; Hervan, E.M.; Mohammadi, S.A.; Moradi, F.; Tabatabaee, M.T.; Pirseyedi, S.M.; Ebrahimi, M.; Fayaz, F.; Kazemi, M. QTL mapping of yield and yield components under normal and salt-stress conditions in bread wheat (Triticum aestivum L.). Plant Mol. Biol. Rep. 2015, 33, 102-120. [CrossRef]

52. Wen, W.; He, Z.; Gao, F.; Liu, J.; Jin, H.; Zhai, S.; Qu, Y.; Xia, X. A high-density consensus map of common wheat integrating four mapping populations scanned by the 90k SNP array. Front. Plant Sci. 2017, 8, 1389. [CrossRef] [PubMed]

53. Zhang, P.; Yin, G.; Zhou, Y.; Qi, A.; Gao, F.; Xia, X.; He, Z.; Li, Z.; Liu, D. QTL mapping of adult-plant resistance to leaf rust in the wheat cross Zhou $8425 \mathrm{~b} /$ Chinese spring using high-density SNP markers. Front. Plant Sci. 2017, 8, 793. [CrossRef] [PubMed]

54. Zhang, C.L.; Jian, J.T.; Feng, J.; Cui, Z.X.; Xu, X.; Sun, D.J. QTL identification for awn length based on 90k array mapping in wheat. Sci. Agric. Sin. 2018, 1, 17-25.

55. Zhao, M.; Wang, G.; Leng, Y.; Wanjugi, H.; Xi, P.; Grosz, M.; Mergoum, M.; Zhong, S. Molecular mapping of fusarium head blight resistance in the spring wheat line ND2710. Phytopathology 2018, in press. [CrossRef] [PubMed]

56. Chao, S.; Zhang, W.; Akhunov, E.; Sherman, J.; Ma, Y.; Luo, M.C.; Dubcovsky, J. Analysis of gene-derived SNP marker polymorphism in us wheat (Triticum aestivum L.) cultivars. Mol. Breed. 2009, 23, 23-33. [CrossRef]

57. Colasuonno, P.; Gadaleta, A.; Giancaspro, A.; Nigro, D.; Giove, S.; Incerti, O.; Mangini, G.; Signorile, A.; Simeone, R.; Blanco, A. Development of a high-density SNP-based linkage map and detection of yellow pigment content QTLs in durum wheat. Mol. Breed. 2014, 34, 1563-1578. [CrossRef]

58. Cui, F.; Li, J.; Ding, A.; Zhao, C.; Wang, L.; Wang, X.; Li, S.; Bao, Y.; Li, X.; Feng, D. Conditional QTL mapping for plant height with respect to the length of the spike and internode in two mapping populations of wheat. Theor. Appl. Genet. 2011, 122, 1517-1536. [CrossRef] [PubMed]

59. Kato, K.; Miura, H.; Sawada, S. Mapping QTLs controlling grain yield and its components on chromosome 5a of wheat. Theor. Appl. Genet. 2000, 101, 1114-1121. [CrossRef]

60. Huang, X.Q.; Kempf, H.; Ganal, M.W.; Röder, M.S. Advanced backcross QTL analysis in progenies derived from a cross between a german elite winter wheat variety and a synthetic wheat (Triticum aestivum L.). Theor. Appl. Genet. 2004, 109, 933-943. [CrossRef] [PubMed]

61. Quarrie, S.A.; Steed, A.; Calestani, C.; Semikhodskii, A.; Lebreton, C.; Chinoy, C.; Steele, N.; Pljevljakusić, D.; Waterman, E.; Weyen, J. A high-density genetic map of hexaploid wheat (Triticum aestivum L.) from the cross Chinese spring $\times$ SQ1 and its use to compare QTLs for grain yield across a range of environments. Theor. Appl. Genet. 2005, 110, 865-880. [CrossRef] [PubMed]

62. Wang, J.; Liu, W.; Wang, H.; Li, L.; Wu, J.; Yang, X.; Li, X.; Gao, A. QTL mapping of yield-related traits in the wheat germplasm 3228. Euphytica 2011, 177, 277-292. [CrossRef] 
63. Kirigwi, F.M.; Van, G.M.; Brownguedira, G.; Gill, B.S.; Paulsen, G.M.; Fritz, A.K. Markers associated with a QTL for grain yield in wheat under drought. Mol. Breed. 2007, 20, 401-413. [CrossRef]

64. Liu, L.H.; Wang, L.X.; Ji, Y.; Zheng, Y.L.; Zhao, C.P. Association mapping of six agronomic traits on chromosome 4A of wheat (Triticum aestivum L.). Mol. Plant Breed. 2010, 1, 5. [CrossRef]

65. Groos, C.; Robert, N.; Bervas, E.; Charmet, G. Genetic analysis of grain protein-content, grain yield and thousand-kernel weight in bread wheat. Theor. Appl. Genet. 2003, 106, 1032-1040. [CrossRef] [PubMed] 